\title{
Design of UDE-based Dynamic Surface Control for Dynamic Positioning of Vessels with Complex Disturbances and Input \\ Constraints
}

\author{
Youqiang Huang ${ }^{1}$, Defeng $\mathrm{Wu}^{1,2^{*}}$, Zibin Yin ${ }^{1,2}$ and Zhi-Ming Yuan ${ }^{3}$
}

Addresses: 1. School of Marine Engineering, Jimei University, Xiamen 361021, PRC

2. Fujian Provincial Key Laboratory of Naval Architecture and Ocean

Engineering, Xiamen 361021, PRC

3. Department of Naval Architecture, Ocean \& Marine Engineering,

University of Strathclyde, Glasgow, G4 0LZ, United Kingdom

\begin{abstract}
In practice, dynamic positioning (DP) vessels are subjected to complex disturbances as well as the magnitude and changing rate constraints of the thrusts and moments. This study applied a dynamic surface controller based on an uncertainty and disturbance estimator (UDE) to a DP vessel with complex disturbances and input constraints. The UDE was designed to estimate and handle the complex disturbances. An auxiliary dynamic system (ADS) and smooth switching function were employed to compensate for the input constraints and avoid the singularity phenomenon caused by the ADS, respectively. The combination of the UDE method and dynamic surface control (DSC) technology significantly simplified the design process for the control law and increased the practicability for DP vessels. The stability of the proposed control law was proved using the Lyapunov theory. The effectiveness of the control law and possibility of actually applying it to a DP vessel were verified using simulation experiments.
\end{abstract}

Keywords: Uncertainty and disturbance estimator (UDE); Input 
constraints; Dynamic positioning of vessels; Dynamic surface controller; Auxiliary dynamic system (ADS)

\section{Introduction}

Dynamic positioning systems (DPS) as an important equipment for the off-shore operations, which have been widely used in oil and gas exploitation, the laying and dredging of submarine pipelines, and the investigation of maritime accidents [1-3]. The DPS is mainly composed of a basic thrust allocation and propulsion subsystem, an auxiliary measurement subsystem, and a crucial control subsystem. In marine environment with complex disturbances, a dynamic positioning (DP) vessel can maintain the desired position or accurately move along the desired trajectory, relying on the forces and moments generated by its own thrust allocation and propulsion systems [4-6]. As an essential part of the DPS, a superior control subsystem is the basis of a DP vessel's precise positioning or trajectory tracking. Thus, the study of the control strategy for DPS has aroused the enthusiasm of a large number of researchers.

As a representative of early control technology, proportional integral derivative (PID) control technology was first applied to a DPS in the 1960s [7]. Based on the inherent nonlinearity of a DP vessel, a nonlinear PID (NLPID) control method was proposed in [8], which enhanced the self-adaptability and robustness of the control subsystem. Motivated by the practical engineering requirements, an output control strategy based on Kalman filtering and optimal theory was proposed by Balchen et al. [9], which was quickly applied to the DP control subsystem [10-12]. With the development of the nonlinear control theory and its correlated technologies, numerous advanced nonlinear control methods have been successively used for the control of a DPS, including sliding mode control (SMC) [13-15], adaptive fuzzy control[16,17], neural network (NN) [18,19], and active disturbance rejection control (ADRC) [20]. Parameter optimization, DO 
and $\mathrm{NN}$ estimator are not embedded in these aforementioned methods. Thus, they have limitations when estimating complex disturbances [21]. To address these problems, $\mathrm{Hu}$ et al. [22] proposed the dynamic surface control (DSC) technology based on a disturbance observer (DO), which the DO was designed to estimate the unknown environmental disturbances of a DP vessel. A NN controller based on the vectorial backstepping technique was proposed in [23]. The modelling uncertainties were compensated by radial basis function (RBF) networks. The control parameters of ADRC were optimised using the adaptive hybrid biogeography-based optimisation and differential evolution (AHBBODE) optimisation algorithm in [21], which improved the ability of ADRC to handle complex internal and external disturbances. In order to compensate the modelling uncertainties and external disturbances, an adaptive SMC method based on NN and DO was proposed in [24]. However, these previously mentioned control methods gave little attention to the problem of the thrust magnitude and rate constraints caused by the inherent structure of the propeller. In practical engineering applications, the maximum power provided by thrusters is limited. The cost of the whole system will be greatly increased by increasing the maximum power, which will significantly decrease the economic benefits of the device. It is important to mitigate the impact of the actuator constraints. Du et al.[25] proposed the DSC method based on DO, which was used to design the controller of a DP vessel with disturbances under input saturation. An auxiliary dynamic system (ADS) was introduced to mitigate the impact of input saturation. However, the modelling uncertainties and thrust rate limitation were neglected. The NN-based DSC law was proposed to design the controller of DP vessels under input constraints in [26] by $\mathrm{Hu}$ et al. The unknown dynamic is approximated by designed NN. The error-driven-based adaptive NN technique was proposed in [27]. The modelling uncertainties are accurately estimated in this method, and the actual input saturation are taken into 
consideration, but the unknown disturbances connot be accurately estimated and compensated. Zhong et al. [28] proposed the UDE method, which could be directly used for system control and the estimation of external disturbances and modelling uncertainties. The UDE was used in general system control to estimate disturbances and uncertainties, which achieved a good robust performance in [29]. The UDE was also applied to industrial processes in [30]. The UDE can estimate complex internal and external disturbances, with an excellent estimation performance and simplified controller structure. In [31], the UDE was utilised in a magnetic levitation system because of its superior ability to estimate complex disturbances. These examples of the UDE was used in three different situations show that it has an excellent estimation performance for disturbances and modelling uncertainties, as well as a simple design. Although the UDE has been widely studied and applied to many different systems, there is almost no information about its application to a DPS. Therefore, this study applied the UDE to a DPS for the estimation of complex disturbances. Considering the problem of the input constraints caused by the inherent structure of propellers in engineering practice, the ADS was used to handle the input constraints, and the smooth switching function was introduced to avoid the singularity problem caused by the ADS. This paper proposes a UDE-based DSC method for the controller design of the DP vessel with complex disturbances and input constraints. The main contributions of this study are summarised as follows.

(1).A UDE was designed to handle the unknown disturbances and modelling uncertainties of a DP vessel.

(2).An ADS was established to improve the nonlinearity of the input constraints, and a smooth switching function was designed to avoid the singularity problem caused by the ADS.

(3). The proposed UDE-based DSC scheme simultaneously considers the input magnitude and rate constraints as well as complex disturbances, the 
ADS and smooth switch function handle the input constraints and singularity phenomenon, respectively.

The rest of the paper is organised as follows. A description of the mathematical model of a vessel with the DPS and the preliminary work is given in Section 2. Section 3 introduces the design of the UDE used for a DP vessel, and introduces the smooth transition function and ADS. In addition, the design of the UDE-based dynamic surface controller is discussed, along with a stability analysis. Section 4 shows how the proposed control strategy was verified using simulations. The conclusions are drawn in Section 5.

\section{Mathematical model of DP vessels}

A DPS is mainly composed of the position and attitude measurement module, control module, and propulsion module (thrusters and propulsion subsystem)[4]. A structural schematic diagram of a dynamic surface controller based on the UDE is illustrated in Fig.1. The mathematical model of a DP vessel is divided into a kinematic model and dynamic model, which are described as follows.

\subsection{Kinematic model of DP vessel}

Due to the vessel moves on the water, and the speed is relatively low, the motion of the vessel can be simplified to three degrees of freedom motion on the horizontal plane. Two right-rand coordinate systems are given in Fig.2 for building the mathematical model of the vessel [32]. $O X_{0} Y_{0} Z_{0}$ is the inertial frame (IF), which is also known as the earth-fixed frame (EF). The origin $O$ is located at a fixed point on the earth. The $O X_{0}$ axis, $O Y_{0}$ axis, and $O Z_{0}$ point toward the north, east, and earth's core, respectively. $A X Y Z$ is a body-fixed frame (BF) with the vessel's centre of gravity as the origin $A$. The axes $A X, A Y$, and $A Z$ are directed from the origin $A$ toward the fore, starboard, and bottom directions, respectively. The position and heading 
are described in the $\mathrm{EF}$ as $\eta(x, y, \psi)$. The velocity is described in the $\mathrm{BF}$ as $v(u, v, r)$. The kinematic model is described as follows [32]:

$$
\dot{\eta}=J(\psi) v
$$

where $\eta=\left[\begin{array}{lll}x & y & \psi\end{array}\right]^{T}$ is a vector consisting of the vessel actual position $(x, y)$ and the yaw angle $\psi \in\left[\begin{array}{ll}0 & 2 \pi\end{array}\right]$.In addition, $v=\left[\begin{array}{lll}u & v & r\end{array}\right]^{T}$ represents the vector consisting of the vessel's movement or rotation velocity along the $A X$ axis, $A Y$ axis, and $A Z$ axis, respectively. The coordinate system transformation matrix can be written as follows: $J(\psi)=\left[\begin{array}{ccc}\cos (\psi) & -\sin (\psi) & 0 \\ \sin (\psi) & \cos (\psi) & 0 \\ 0 & 0 & 1\end{array}\right]$, where $J^{T}(\psi)=J^{-1}(\psi)$ and $\|J(\psi)\|=1$.

\subsection{Dynamic model of DP vessel}

The nonlinear dynamic equation of a DP vessel with complex disturbances can be written as follows [32]:

$$
M \dot{v}+C(v) v+D v+\Delta_{f}=\tau+d
$$

where $M \in R^{3 \times 3}$ is a matrix composed of the vessel's weight inertia and additional hydrodynamic inertia; $C(v) \in R^{3 \times 3}$ is a Coriolis centripetal matrix; $D \in R^{3 \times 3}$ represents the linear hydrodynamic damping parameter matrix; and $\Delta_{f}=\left[\begin{array}{lll}\Delta_{f 1} & \Delta_{f 2} & \Delta_{f 3}\end{array}\right]^{T}$ is a vector composed of the modelling uncertainties of the vessel. $\tau=\left[\begin{array}{lll}\tau_{1} & \tau_{2} & \tau_{3}\end{array}\right]^{T}$ is the control forces and moments vector provided by the propulsion module. In practice, because of the inherent structure of the propeller, the control forces and moments are limited by the magnitude and rate, which can be expressed as follows:

$$
\tau_{p i}=\left\{\begin{array}{ccc}
\tau_{M i} & \text { if } & \tau_{c i}>\tau_{M i} \\
\tau_{c i} & \text { if } & -\tau_{M i} \leq \tau_{c i} \leq \tau_{M i} \\
-\tau_{M} & \text { if } & \tau_{c i}<-\tau_{M i}
\end{array},\left|\dot{\tau}_{i}\right| \leq \tau_{R i}\right.
$$

where $\tau_{M i}>0(i=1,2,3)$ is the maximum control forces or moments that 
can be provided by the propulsion module; $\tau_{R i}>0(i=1,2,3)$ denotes the maximum control input rate; $\tau_{c}=\left[\begin{array}{lll}\tau_{c 1} & \tau_{c 2} & \tau_{c 3}\end{array}\right]^{T}$ is the command control input vector; $\tau_{p}=\left[\begin{array}{ccc}\tau_{p 1} & \tau_{p 2} & \tau_{p 3}\end{array}\right]^{T}$ is the control input vector with magnitude limitations; $d=\left[\begin{array}{lll}d_{1} & d_{2} & d_{3}\end{array}\right]^{T}$ is the unknown disturbances vector, which are caused by the wind, waves, and current in the body-fixed frame.

Assumption 1: The changing rate of ocean environmental disturbance is bounded [25], and there is an unknown positive constant $\varepsilon$ such that $\|\dot{d}(t)\| \leq \varepsilon<\infty$.

Assumption 2: The modelling uncertainty of vessel $\Delta_{f}$ is unknown and yet bounded, with finite changing rates, and there exists an unknown positive constant $\sigma_{\text {such that }}\left\|\dot{\Delta}_{f}\right\| \leq \sigma<\infty$.

Remark 1: The disturbance from the wind, waves and current in a marine environment is constantly changing and is difficult to predict, but its total energy is limited. Therefore, the disturbance of a vessel can be considered to be unknown but bounded, and the rate of change of this disturbance is also bounded. Thus, Assumption 1 is reasonable.

Remark 2: The modelling uncertainty is unknown and varies with the motion state of the vessel. Because the speed of the vessel is limited, the modelling uncertainty can be considered to be unknown but bounded, and the change rate of the modelling uncertainty is also bounded. Therefore, Assumption 2 is reasonable.

Remark 3: According to Assumptions 1 and 2, the rate of change of a vessel's external environment disturbance is bounded, as is the rate of change of the modelling uncertainty is bounded. Therefore, the rate of change of the complex disturbance is bounded [33], and there exists an unknown positive constant $U_{d}^{*}$ such that: 


$$
\left\|\dot{d}(t)-\dot{\Delta}_{f}\right\| \leq \varepsilon+\sigma \leq U_{d}^{*}
$$

\section{Design of nonlinear dynamic surface controller based on}

\section{UDE for DP vessel}

\subsection{Designing UDE for DP vessel}

In this subsection, the UDE was established to estimate the complex disturbances of a DP vessel, and the stability of the UDE was analysed.

According to equation (2), the complex disturbances of a DP vessel can be expressed as follows:

$$
U_{d}=-\Delta_{f}+d=M \dot{v}+C(v) v+D v-\tau
$$

where $U_{d}=\left(-\Delta_{f}+d\right) \in R^{3}$ is a complex disturbances vector composed of the sum of the modelling uncertainties and environmental disturbances of the vessel.

The UDE of a DP vessel can be given as follows:

$$
\hat{U}_{d}=\left(-\Delta_{f}+d\right) \star g_{f}(t)=U_{d} \star g_{f}(t)=(M \dot{v}+C(v) v+D v-\tau) \star g_{f}(t)
$$

where $\hat{U}_{d} \in R^{3}$ denotes the estimate of $U_{d}$, “ $\star$ " denotes the convolution operator, and $g_{f}(t)$ denotes the impulse response of a filter, covering the spectrum of $U_{d}$.

The complex disturbance estimation error vector $\widetilde{U}_{d} \in R^{3}$ can be defined by the UDE as follows:

$$
\widetilde{U}_{d}=U_{d}-\hat{U}_{d}
$$

Equations (5), (6), and (7) can be represented in the $s$-domain as follows:

$$
\begin{gathered}
U_{D}(s)=-\Delta_{f}(s)+D(s) \\
\hat{U}_{D}(s)=G_{f}(s)\left[-\Delta_{f}(s)+D(s)\right] \\
\widetilde{U}_{D}(s)=U_{D}(s)-\hat{U}_{D}(s)
\end{gathered}
$$


where $G_{f}(s)=\operatorname{diag}\left[G_{f 1}(s) \quad G_{f 2}(s) \quad G_{f 3}(s)\right]$ denotes the filter matrix, and the filter $G_{f i}(s)=\frac{q_{i}}{s+q_{i}}\left(q_{i}>0, i=1,2,3\right)$. More details about the design of a UDE filter are available in [34].

Substituting equations (8) and (9) into equation (10) produces the following:

$$
\widetilde{U}_{D}(s)=\left(I-G_{f}(s)\right) U_{D}(s)
$$

where, $\quad \widetilde{U}_{D}(s)=\left[\begin{array}{lll}\widetilde{U}_{D 1}(s) & \widetilde{U}_{D 2}(s) & \widetilde{U}_{D 3}(s)\end{array}\right]^{T}, U_{D}(s)=\left[\begin{array}{lll}U_{D 1}(s) & U_{D 2}(s) & U_{D 3}(s)\end{array}\right]^{T}$, $\hat{U}_{D}(s)=\left[\begin{array}{lll}\hat{U}_{D 1}(s) & \hat{U}_{D 2}(s) & \hat{U}_{D 3}(s)\end{array}\right]^{T}$, and $I \in R^{3 \times 3}$ denotes the third-order identity matrix.

Thus, equation (11) can be rewritten as follows:

$$
\widetilde{U}_{D i}(s)=\left(1-\frac{q_{i}}{s+q_{i}}\right) U_{D i}(s)=\frac{s}{s+q_{i}} U_{D i}(s)
$$

This can be simplified as follows:

$$
\begin{gathered}
\left(s+q_{i}\right) \widetilde{U}_{D i}(s)=s U_{D i}(s) \\
s \widetilde{U}_{D i}(s)=-q_{i} \widetilde{U}_{D i}(s)+s U_{D i}(s)
\end{gathered}
$$

After the inverse Laplace transform, equation (14) can be rewritten as follows:

$$
\dot{\widetilde{U}}_{d}=-q \widetilde{U}_{d}+\dot{U}_{d}
$$

where $q=\operatorname{diag}\left(q_{1} \quad q_{2} q_{3}\right)$.

The UDE Lyapunov function candidates can be selected as follows:

$$
V_{U_{d}}=\frac{1}{2} \widetilde{U}_{d}{ }^{T} \widetilde{U}_{d}
$$

In light of equation (15), the time derivative of equation (16) is written as follows:

$$
\dot{V}_{U_{d}}=\widetilde{U}_{d}^{T} \dot{\widetilde{U}}_{d}=-\widetilde{U}_{d}{ }^{T} q \widetilde{U}_{d}+\widetilde{U}_{d}{ }^{T} \dot{U}_{d}
$$

According to equation (4) and Young's inequality, we have the following: 


$$
\widetilde{U}_{d}{ }^{T} \dot{U}_{d} \leq \mu \widetilde{U}_{d}{ }^{T} \widetilde{U}_{d}+\frac{U_{d}^{*}}{4 \mu}
$$

where $\mu$ is a positive constant. Substituting equations (16) and (18) into equation (17) produces the following:

$$
\dot{V}_{U_{d}} \leq-\widetilde{U}_{d}{ }^{T} q \widetilde{U}_{d}+\mu \widetilde{U}_{d}{ }^{T} \widetilde{U}_{d}+\frac{U_{d}^{* 2}}{4 \mu} \leq-2\left(\lambda_{\min }(q)-\mu\right) V_{U_{d}}+\frac{U_{d}^{*}}{4 \mu} \leq-2 \beta_{0} V_{U_{d}}+\gamma_{0}
$$

where $\beta_{0}=\left(\lambda_{\min }(q)-\mu\right)>0$, and $\lambda_{\min }(\cdot)$ denotes the minimum eigenvalue of a matrix, $\gamma_{0}=\frac{U_{d}^{* 2}}{4 \mu}>0$. Thus, $q$ must satisfy $\lambda_{\min }(q)>\mu$.

Theorem 1: Under Assumption 1 and Assumption 2, the estimation error vector $\widetilde{U}_{d}=\widetilde{d}-\widetilde{\Delta}_{f}$ of the complex disturbances for the DP vessel by the UDE, settles with in set $\Omega_{\widetilde{U}_{d}}=\left\{\widetilde{U}_{d} \in R^{3}\|\| \widetilde{U}_{d} \| \leq \xi_{\widetilde{U}_{d}}, \xi_{\widetilde{U}_{d}}>\sqrt{\frac{\gamma_{0}}{\beta_{0}}}\right\}$. Under the condition of $q_{i}>\mu$, by appropriately adjusting filter values $q_{i}$ and $\mu$, the value of $\left\|\widetilde{U}_{d}\right\|$ can be made arbitrarily small. Thus, the UDE can estimate the complex disturbances of a DP vessel with arbitrary precision, and the estimation error is arbitrarily small.

Proof: Solving inequality (19) gives the following:

$$
0 \leq V_{U_{d}}(t) \leq \frac{\gamma_{0}}{2 \beta_{0}}+\left(V_{U_{d}}(0)-\frac{\gamma_{0}}{2 \beta_{0}}\right) e^{-2 \beta_{0} t}
$$

Obviously, $V_{U_{d}}(t)$ is bounded. Integrating equations (16) and (20) gives the following:

$$
0 \leq\left\|\widetilde{U}_{d}\right\| \leq \sqrt{\frac{\gamma_{0}}{\beta_{0}}+2\left[V_{U_{d}}(0)-\frac{\gamma_{0}}{2 \beta_{0}}\right] e^{-2 \beta_{0} t}}
$$

It can be seen from equation $(21)$ that for any positive constant $\xi_{\widetilde{U}_{d}}>\sqrt{\frac{\gamma_{0}}{\beta_{0}}}$, 
there exists a time constant, $T_{\widetilde{U}_{d}}$, such that $\left\|\widetilde{U}_{d}\right\| \leq \xi_{\widetilde{U}_{d}}$ for all $t>T_{\widetilde{U}_{d}}$. Therefore, the estimation error vector, $\widetilde{U}_{d}=\widetilde{d}-\widetilde{\Delta}_{f}$, of the complex disturbances for the DP vessel by the UDE settles within compact set $\Omega_{\widetilde{U}_{d}}=\left\{\widetilde{U}_{d} \in R^{3} \mid\left\|\widetilde{U}_{d}\right\| \leq \xi_{\widetilde{U}_{d}}, \xi_{\widetilde{U}_{d}}>\sqrt{\frac{\gamma_{0}}{\beta_{0}}}\right\}$. Under the condition of $q_{i}>\mu$, by appropriately adjusting filter values $q_{i}$ and $\mu$, the values of $\left\|\widetilde{U}_{d}\right\|$ can be made arbitrarily small. Thus, the UDE can estimate the complex disturbances of a DP vessel with arbitrary precision, and the estimation error is arbitrarily small. Thus, theorem 1 holds.

\subsection{Dynamic surface controller design based on UDE}

This subsection, the DSC strategy and the UDE are synthesized to design control law, which can achieve accurately motion control for a DP vessel with complex disturbances, input magnitude and rate constraints. An ADS is employed to handle the problem of the input constraints [35], and a smooth switching function [36] is adopted to avoid the singularity phenomenon produced by the ADS. The control law is designed as follows:

Step 1: Define the vessel's position and yaw angle error vector $\vartheta_{1} \in R^{3}$ as follows:

$$
\vartheta_{1}=\eta-\eta_{d}
$$

where $\eta_{d}=\left[\begin{array}{lll}x_{d} & y_{d} & \psi_{d}\end{array}\right]^{T}$ denotes the vector consists of the desired position $\left(x_{d}, y_{d}\right)$ and yaw angle $\psi_{d}$ vector in the EF.

In the light of equation (1), the time derivative of equation (22) is found as follows:

$$
\dot{\vartheta}_{1}=J(\psi) v-\dot{\eta}_{d}
$$

Virtual control vector $\alpha_{1} \in R^{3}$ for $v$ is designed as follows: 


$$
\alpha_{1}=J^{-1}(\psi)\left(-\Gamma_{1} \vartheta_{1}+\dot{\eta}_{d}\right)
$$

where $\Gamma_{1} \in R^{3 \times 3}$ is the designed positive definite parameter diagonal matrix.

Let $v_{d} \in R^{3}$ as the output of the first-order low-pass filter of the virtual control vector $\alpha_{1}$ :

$$
T \dot{v}_{d}+v_{d}=\alpha_{1}, v_{d}(0)=\alpha_{1}(0)
$$

where $v_{d} \in R^{3}$ is the state vector of the filter, and $T>0$ is the filter time constant.

Remark 4: The simple algebraic calculation $\dot{v}_{d}=\left(\alpha_{1}-v_{d}\right) / T$ can be used instead of the derivative operation of the virtual control vector, a differential explosion is avoided. Thus, the design of the control law is simplified and easy to apply in practical engineering.

Step 2: Define the vessel velocity error vector, $\vartheta_{2} \in R^{3}$, as follows:

$$
\vartheta_{2}=v-v_{d}
$$

According to equations (2), (25), and (26), we have the following:

$$
M \dot{\vartheta}_{2}=-C(v) v-D v+\tau+d-\Delta_{f}-M \frac{\alpha_{1}-v_{d}}{T}
$$

Filter error vector $\delta \in R^{3}$ can be defined as follows:

$$
\delta=v_{d}-\alpha_{1}
$$

In order to handle the input constraint problem, an $\operatorname{ADS}[36]$ is designed as follows:

$$
\dot{\chi}_{i}=-\Phi_{\chi_{i}} \chi_{i}-\frac{\left|\vartheta_{2 i} \Delta \tau_{a i}\right|+0.5 \rho_{i}^{2} \Delta \tau_{a i}^{2}}{\chi_{i}} h\left(\chi_{i}\right)+\rho_{i} \Delta \tau_{a i}
$$

where $\Phi_{\chi_{i}}>1, \quad \rho_{i}>0, \quad \rho=\operatorname{diag}\left(\rho_{1} \quad \rho_{2} \quad \rho_{3}\right), \Delta \tau_{a i}=\tau_{p i}-\tau_{c i}, \quad(i=1,2,3)$, and $\Delta \tau_{a}=\tau_{p}-\tau_{c}=\left[\begin{array}{lll}\Delta \tau_{a 1} & \Delta \tau_{a 2} & \Delta \tau_{a 3}\end{array}\right]^{T}, \chi_{i}$ is the state of the ADS.

Remark 5: The ADS depended on $\Delta \tau_{a i}$ and $h\left(\chi_{i}\right)$, if they are not chosen properly, a singularity phenomenon will occur in the ADS. 
The smooth switching function $h\left(\chi_{i}\right)$ is constructed as follows:

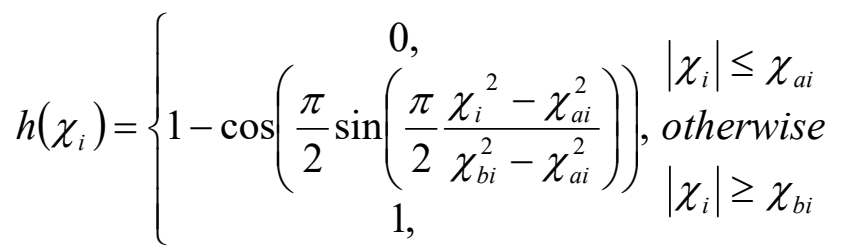

where $0<\chi_{a i}<\chi_{b i}(i=1,2,3)$, and $\chi_{b i}$ is a positive design constant. Function $h\left(\chi_{i}\right)$ is introduced to avoid the singularity of the $\operatorname{ADS}$ of equation (29) when $\chi_{i}$ approaches zero [36].

Lemma 1 [37]: Function $h\left(\chi_{i}\right)$ is a smooth switching function for all $\chi_{i}$. Proof See Appendix A.

To compensate the input constraint problem, let the commanded control input vector, $\tau_{c} \in R^{3}$, as follows:

$$
\tau_{c}=\tau+\Phi_{s} \chi
$$

where $\Phi_{s}=\Phi_{s}{ }^{T} \in R^{3 \times 3}$ is the designed positive definite parameter matrices, and $\chi=\left[\begin{array}{lll}\chi_{1} & \chi_{2} & \chi_{3}\end{array}\right]^{T}$ is the state vector of the ADS.

Replacing equation (5) with equation (6), and substituting equation (6) into equation (27), Let $-\Gamma_{2} \vartheta_{2}=M \dot{\vartheta}_{2}$ the DP vessel's UDE-based DSC law is designed as follows:

$$
\tau_{c}=-\Gamma_{2} \vartheta_{2}+C(v) v+D v+M \frac{\alpha_{1}-v_{d}}{T}+\Phi_{s} \chi-\hat{U}_{d}
$$

where $\Gamma_{2}=\Gamma_{2}{ }^{T} \in R^{3 \times 3}$ is the designed parameter matrices.

The Lyapunov function candidate for the whole control system of the DP vessel is selected as follows:

$$
V=\frac{1}{2} \vartheta_{1}^{T} \vartheta_{1}+\frac{1}{2} \vartheta_{2}^{T} M \vartheta_{2}+\frac{1}{2} \delta^{T} \delta+\frac{1}{2} \chi^{T} \chi+\frac{1}{2} \widetilde{U}_{d}{ }^{T} \widetilde{U}_{d}
$$

The derivative of $V$ equation (33) with respect to time is taken as follows:

$$
\dot{V}=\vartheta_{1}^{T} \dot{\vartheta}_{1}+\vartheta_{2}^{T} M \dot{\vartheta}_{2}+\delta^{T} \dot{\delta}+\chi^{T} \dot{\chi}+\widetilde{U}_{d}^{T} \dot{\widetilde{U}}_{d}
$$

According to equations (23), (24), (26), and (28), along with $\|J(\psi)\|=1$ and 
Young's inequality, the following can be obtained:

$$
\begin{aligned}
\vartheta_{1}^{T} \dot{\vartheta}_{1} & =\vartheta_{1}^{T} J(\psi)\left(\vartheta_{2}+v_{d}\right) \\
& =\vartheta_{1}^{T} J(\psi)\left(\vartheta_{2}+\delta-J^{-1}(\psi) \Gamma_{1} \vartheta_{1}\right) \\
& =-\vartheta_{1}^{T} \Gamma_{1} \vartheta_{1}+\vartheta_{1}^{T} J(\psi)\left(\vartheta_{2}+\delta\right) \\
& \leq-\vartheta_{1}^{T} \Gamma_{1} \vartheta_{1}+2 a_{1} \vartheta_{1}^{T} \vartheta_{1}+\frac{\vartheta_{2}^{T} \vartheta_{2}}{4 a_{1}}+\frac{\delta^{T} \delta}{4 a_{1}}
\end{aligned}
$$

where $a_{1}$ is a positive constant.

In light of equations (7), (27), and (32), along with $\tau=\tau_{c}+\Delta \tau_{b}$ and Young's inequality, the following can be obtained:

$$
\begin{aligned}
\vartheta_{2}^{T} M \dot{\vartheta}_{2} & =\vartheta_{2}^{T}\left(-C(v) v-D v+\tau+\left(d-\Delta_{f}\right)-M \frac{\alpha_{1}-v_{d}}{T}\right) \\
& =\vartheta_{2}^{T}\left(-C(v) v-D v+\tau_{c}+\Delta \tau_{b}+\left(d-\Delta_{f}\right)-M \frac{\alpha_{1}-v_{d}}{T}\right) \\
& =\vartheta_{2}^{T}\left(-\Gamma_{2} \vartheta_{2}+\Phi_{S} \chi+\Delta \tau_{b}+\left(d-\Delta_{f}\right)-\hat{U}_{d}\right) \\
& =-\vartheta_{2}^{T} \Gamma_{2} \vartheta_{2}+\vartheta_{2}^{T} \Phi_{S} \chi+\vartheta_{2}^{T} \widetilde{U}_{d}+\vartheta_{2}^{T} \Delta \tau_{b} \\
& \leq-\vartheta_{2}^{T} \Gamma_{2} \vartheta_{2}+2 a_{2} \vartheta_{2}^{T} \vartheta_{2}+\frac{\left(\Phi_{S} \chi\right)^{T} \Phi_{S} \chi}{4 a_{2}}+\frac{\widetilde{U}_{d}{ }^{T} \widetilde{U}_{d}}{4 a_{2}}+\vartheta_{2}^{T} \Delta \tau_{b}
\end{aligned}
$$

where $a_{2}$ is a positive constant.

In view of equations (23), (24), (25), and (28), the time derivative of equation (28) can found as follows:

$$
\dot{\delta}=-\frac{\delta}{T}+\dot{J}^{-1}(\psi) \Gamma_{1} \vartheta_{1}+J^{-1}(\psi) \Gamma_{1} \dot{\vartheta}_{1}-\dot{J}^{-1}(\psi) \dot{\eta}_{d}-J^{-1}(\psi) \ddot{\eta}_{d}
$$

Set $\quad \Omega_{d}=\left\{\left[\begin{array}{lll}\eta_{d}^{T} & \dot{\eta}_{d}^{T} & \ddot{\eta}_{d}^{T}\end{array}\right]^{T}:\left\|\eta_{d}\right\|^{2}+\left\|\dot{\eta}_{d}\right\|^{2}+\left\|\ddot{\eta}_{d}\right\|^{2} \leq \mathrm{H}_{0}\right\} \quad$ and set $\Omega=\left\{\left[\begin{array}{lllll}\vartheta_{1}^{T} & \vartheta_{2}^{T} & \delta^{T} & \chi^{T} & \tilde{U}_{d}{ }^{T}\end{array}\right]^{T}: V \leq \mathrm{H}\right\}$ are compact sets, where $\mathrm{H}_{0}$ and $\mathrm{H}$ are the desired positive constants. Thus, set $\Omega_{d} \times \Omega$ is also a compact set. Discontinuous function $\beta(\cdot)$ can be obtained as follows:

$$
\begin{aligned}
\left\|\dot{\delta}+\frac{\delta}{T}\right\| & =\dot{J}^{-1}(\psi) \Gamma_{1} \vartheta_{1}+J^{-1}(\psi) \Gamma_{1} \dot{\vartheta}_{1}-\dot{J}^{-1}(\psi) \dot{\eta}_{d}-J^{-1}(\psi) \ddot{\eta}_{d} \\
& \leq \beta\left(\vartheta_{1}^{T}, \vartheta_{2}^{T}, \delta^{T}, \chi^{T}, \widetilde{U}_{d}{ }^{T}, \eta_{d}^{T}, \dot{\eta}_{d}^{T}, \ddot{\eta}_{d}^{T}\right)
\end{aligned}
$$

where $\beta($.$) has a maximum value of N_{0}$ in the compact set $\Omega_{d} \times \Omega$. 
Using equations (37) and (38), we obtain the following:

$$
\begin{aligned}
\delta \dot{\delta} & =-\frac{\delta^{T} \delta}{T}+\frac{\delta^{T} \delta}{T}+\delta^{T} \dot{\delta} \\
& =-\frac{\delta^{T} \delta}{T}+\delta^{T}\left(\frac{\delta}{T}+\dot{\delta}\right) \\
& \leq-\frac{\delta^{T} \delta}{T}+b \delta^{T} \delta+\frac{N_{0}^{2}}{4 b}
\end{aligned}
$$

where $b$ is a positive constant.

According to equation (29) and Young's inequality, the following can be obtained:

$$
\begin{aligned}
\chi_{i} \dot{\chi}_{i} & =\chi_{i}\left(-\Phi_{\chi_{i}} \chi_{i}-\frac{\left|\vartheta_{2 i} \Delta \tau_{a i}\right|+0.5 \rho_{i} \Delta \tau_{a i}^{2}}{\chi_{i}} h\left(\chi_{i}\right)+\rho_{i} \Delta \tau_{a i}\right) \\
& =-\Phi_{\chi_{i}} \chi_{i}^{2}-h\left(\chi_{i}\right)\left|\vartheta_{2 i} \Delta \tau_{a i}\right|-\frac{h\left(\chi_{i}\right)}{2} \rho_{i}^{2} \Delta \tau_{a i}^{2}+\rho_{i} \chi_{i} \Delta \tau_{a i} \\
& \leq-\Phi_{\chi_{i}} \chi_{i}^{2}-h\left(\chi_{i}\right)\left|\vartheta_{2 i} \Delta \tau_{a i}\right|+\frac{\chi_{i}^{2}}{2}-\frac{1}{2} \rho_{i}^{2} \Delta \tau_{a i}^{2}\left(1-h\left(\chi_{i}\right)\right)
\end{aligned}
$$

When $\left|\chi_{i}\right| \geq \chi_{b}$ and $h\left(\chi_{i}\right)=1$, inequality (40) can be rewritten as follows:

$\chi_{i} \dot{\chi}_{i} \leq-\left(\Phi_{\chi_{i}}-\frac{1}{2}\right) \chi_{i}^{2}-\left|\vartheta_{2 i} \Delta \tau_{a i}\right|$, such that

$$
\chi^{T} \dot{\chi} \leq-\chi^{T} \Phi_{\chi} \chi+\frac{1}{2} \chi^{T} \chi-\left|\vartheta_{2}^{T} \Delta \tau_{a}\right|
$$

Substituting equations (19), (35), (36), (38), and (41) into equation (34) and using Young's inequality gives the following:

$$
\begin{aligned}
\dot{V} & \leq-\vartheta_{1}^{T} \Gamma_{1} \vartheta_{1}+2 a_{1} \vartheta_{1}^{T} \vartheta_{1}+\frac{\vartheta_{2}^{T} \vartheta_{2}}{4 a_{1}}+\frac{\delta^{T} \delta}{4 a_{1}}-\vartheta_{2}^{T} \Gamma_{2} \vartheta_{2}+2 a_{2} \vartheta_{2}^{T} \vartheta_{2}+\frac{\left(\Phi_{s} \chi\right)^{T} \Phi_{s} \chi}{4 a_{2}} \\
& +\frac{\widetilde{U}_{d}{ }^{T} \widetilde{U}_{d}}{4 a_{2}}+\vartheta_{2}^{T} \Delta \tau_{b}-\frac{\delta^{T} \delta}{T}+b \delta^{T} \delta+\frac{N_{0}{ }^{2}}{4 b}-\chi^{T} \Phi_{\chi} \chi+\frac{1}{2} \chi^{T} \chi-\left|\vartheta_{2}^{T} \Delta \tau_{a}\right|-\widetilde{U}_{d}{ }^{T} q \widetilde{U}_{d} \\
& +\mu \widetilde{U}_{d}{ }^{T} \widetilde{U}_{d}+\frac{U_{d}^{*}}{4 \mu}+\frac{\vartheta_{2}^{T} \vartheta_{2}}{2}+\frac{\left\|\Delta \tau_{b}\right\|^{2}}{2} \\
& \leq-\left[\lambda_{\min }\left(\Gamma_{1}\right)-2 a_{1}\right] \vartheta_{1}^{T} \vartheta_{1}-\left[\lambda_{\min }\left(\Gamma_{2}\right)-2 a_{2}-\frac{1}{4 a_{1}}-\frac{1}{2}\right] \vartheta_{2}^{T} \vartheta_{2}-\left[\frac{1}{T}-b-\frac{1}{4 a_{1}}\right] \delta^{T} \delta \\
& -\left[\lambda_{\min }\left(\Phi_{\chi}-\frac{\Phi_{s}^{T} \Phi_{s}}{4 a_{2}}\right)-\frac{1}{2}\right] \chi^{T} \chi-\left[\lambda_{\min }(q)-\mu\right] \widetilde{U}_{d}{ }^{T} \widetilde{U}_{d}+\frac{N_{0}{ }^{2}}{4 b}+\frac{U_{d}^{*}}{4 \mu}+\frac{\left\|\Delta \tau_{b}\right\|^{2}}{2} \\
& \leq-2 \kappa_{1} V+\theta_{1}
\end{aligned}
$$


where

$\kappa_{1}=\min \left\{\lambda_{\min }\left(\Gamma_{1}\right)-2 a_{1}, \frac{\lambda_{\min }\left(\Gamma_{2}\right)-2 a_{2}-\frac{1}{4 a_{1}}-\frac{1}{2}}{\lambda_{\max }(M)}, \frac{1}{T}-b-\frac{1}{4 a_{1}}, \lambda_{\min }\left(\Phi_{\chi}-\frac{\Phi_{s}^{T} \Phi_{s}}{4 a_{2}}\right)-\frac{1}{2}, \lambda_{\min }(q)-\mu\right\}$

$\lambda_{\max }($.$) denotes the minimum characteristic value of a matrix, and$ $\theta_{1}=\frac{N_{0}^{2}}{4 b}+\frac{U_{d}^{* 2}}{4 \mu}+\frac{\left\|\Delta \tau_{b}\right\|^{2}}{2}$

Otherwise, when $0 \leq h\left(\chi_{i}\right)<1$, noting that $\vartheta_{2 i} \Delta \tau_{a i} \leq \frac{\vartheta_{2 i}^{2}}{2}+\frac{\Delta \tau_{a i}^{2}}{2}$, the following is obtained:

$$
\vartheta_{2}^{T} \Delta \tau_{a} \leq \frac{\vartheta_{2}^{T} \vartheta_{2}}{2}+\frac{\left\|\Delta \tau_{a}\right\|^{2}}{2}
$$

Substituting equations (19), (35), (36), (39), and (40) into equation (34) and using (43) and Young's inequality produces the following: 


$$
\begin{aligned}
& \dot{V} \leq-\vartheta_{1}^{T} \Gamma_{1} \vartheta_{1}+2 a_{1} \vartheta_{1}^{T} \vartheta_{1}+\frac{\vartheta_{2}^{T} \vartheta_{2}}{4 a_{1}}+\frac{\delta^{T} \delta}{4 a_{1}}-\vartheta_{2}^{T} \Gamma_{2} \vartheta_{2}+2 a_{2} \vartheta_{2}^{T} \vartheta_{2}+\frac{\left(\Phi_{S} \chi\right)^{T} \Phi_{S} \chi}{4 a_{2}} \\
& +\frac{\widetilde{U}_{d}^{T} \widetilde{U}_{d}}{4 a_{2}}+\vartheta_{2}^{T} \Delta \tau_{b}-\frac{\delta^{T} \delta}{T}+b \delta^{T} \delta+\frac{N_{0}^{2}}{4 b}+\frac{1}{2} \chi^{T} \chi-\chi^{T} \Phi_{\chi} \chi-\sum_{i=1}^{3} \frac{\left|\vartheta_{2 i} \Delta \tau_{a i}\right| h\left(\chi_{i}\right)}{2} \\
& +\sum_{i=1}^{3} \frac{\rho_{i}^{2} \Delta \tau_{a i}^{2}\left[1-h\left(\chi_{i}\right)\right]}{2}-\widetilde{U}_{d}{ }^{T} q \widetilde{U}_{d}+\mu \widetilde{U}_{d}{ }^{T} \widetilde{U}_{d}+\frac{U_{d}^{* 2}}{4 \mu} \\
& \leq-\vartheta_{1}^{T} \Gamma_{1} \vartheta_{1}+2 a_{1} \vartheta_{1}^{T} \vartheta_{1}+\frac{\vartheta_{2}^{T} \vartheta_{2}}{4 a_{1}}+\frac{\delta^{T} \delta}{4 a_{1}}-\vartheta_{2}^{T} \Phi_{2} \vartheta_{2}+2 a_{2} \vartheta_{2}^{T} \vartheta_{2}+\frac{\left(\Phi_{S} \chi\right)^{T} \Phi_{S} \chi}{4 a_{2}} \\
& +\frac{\widetilde{U}_{d}^{T} \widetilde{U}_{d}}{4 a_{2}}+\vartheta_{2}^{T} \Delta \tau_{b}-\frac{\delta^{T} \delta_{2}}{T}+b \delta^{T} \delta+\frac{N_{0}{ }^{2}}{4 b}+\frac{1}{2} \chi^{T} \chi-\chi^{T} \Phi_{\chi} \chi+\frac{\left(\rho \Delta \tau_{a}\right)^{T} \rho \Delta \tau_{a}}{2}-\widetilde{U}_{d}^{T} q \widetilde{U}_{d} \\
& +\mu \widetilde{U}_{d}^{T} \widetilde{U}_{d}+\frac{U_{d}^{* 2}}{4 \mu} \\
& \leq-\vartheta_{1}^{T} \Gamma_{1} \vartheta_{1}+2 a_{1} \vartheta_{1}^{T} \vartheta_{1}+\frac{\vartheta_{2}^{T} \vartheta_{2}}{4 a_{1}}+\frac{\delta^{T} \delta}{4 a_{1}}-\vartheta_{2}^{T} \Gamma_{2} \vartheta_{2}+2 a_{2} \vartheta_{2}^{T} \vartheta_{2}+\frac{\left(\Phi_{s} \chi\right)^{T} \Phi_{s} \chi}{4 a_{2}} \\
& +\frac{\widetilde{U}_{d}{ }^{T} \widetilde{U}_{d}}{4 a_{2}}+\frac{\vartheta_{2}^{T} \vartheta_{2}}{2}+\frac{\Delta \tau_{b}^{T} \Delta \tau_{b}}{2}-\frac{\delta^{T} \delta}{T}+b \delta^{T} \delta+\frac{N_{0}{ }^{2}}{4 b}-\chi^{T} \Phi_{\chi} \chi+\frac{\left(\rho \Delta \tau_{a}\right)^{T} \rho \Delta \tau_{a}}{2}-\widetilde{U}_{d}{ }^{T} q \widetilde{U}_{d} \\
& +\mu \widetilde{U}_{d}^{T} \widetilde{U}_{d}+\frac{U_{d}^{* 2}}{4 \mu} \\
& \leq-\left[\lambda_{\min }\left(\Gamma_{1}\right)-2 a_{1}\right] \vartheta_{1}^{T} \vartheta_{1}-\left[\lambda_{\min }\left(\Gamma_{2}\right)-2 a_{2}-\frac{1}{4 a_{1}}-\frac{1}{2}\right] \vartheta_{2}^{T} \vartheta_{2}-\left[\frac{1}{T}-b-\frac{1}{4 a_{1}}\right] \delta^{T} \delta \\
& -\left[\lambda_{\min }\left(\Phi_{\chi}-\frac{\Phi_{s}^{T} \Phi_{s}}{4 a_{2}}\right)-\frac{1}{2}\right] \chi^{T} \chi-\left[\lambda_{\min }(q)-\mu\right] \widetilde{U}_{d}^{T} \widetilde{U}_{d}+\frac{N_{0}{ }^{2}}{4 b}+\frac{U_{d}^{* 2}}{4 \mu}+\frac{\left\|\Delta \tau_{b}\right\|^{2}}{2}+\frac{\left\|\rho \Delta \tau_{a}\right\|^{2}}{2} \\
& \leq-2 \kappa_{2} V+\theta_{2}
\end{aligned}
$$

where

$\kappa_{2}=\min \left\{\lambda_{\min }\left(\Gamma_{1}\right)-2 a_{1}, \frac{\lambda_{\min }\left(\Gamma_{2}\right)-2 a_{2}-\frac{1}{4 a_{1}}-\frac{1}{2}}{\lambda_{\max }(M)}, \frac{1}{T}-b-\frac{1}{4 a_{1}}, \lambda_{\min }\left(\Phi_{\chi}-\frac{\Phi_{s}^{T} \Phi_{s}}{4 a_{2}}\right)-\frac{1}{2}, \lambda_{\min }(q)-\mu\right\}$, and $\theta_{2}=\frac{N_{0}{ }^{2}}{4 b}+\frac{U_{d}^{*^{2}}}{4 \mu}+\frac{\left\|\Delta \tau_{b}\right\|^{2}}{2}+\frac{\left\|\rho \Delta \tau_{a}\right\|^{2}}{2}$.

Combining equations (42) and (44) produces the following:

$$
\dot{V} \leq-2 \kappa V+\theta
$$

where $\kappa=\min \left\{\kappa_{1}, \kappa_{2}\right\}$ and $\theta=\max \left\{\theta_{1}, \theta_{2}\right\}$. Because $\theta, a_{1}, a_{2}, b, \mu>0$ is required, the following controller parameter conditions should be satisfied: 


$$
\begin{gathered}
\lambda_{\text {min }}\left(\Gamma_{1}\right)>2 a_{1} \\
\lambda_{\text {min }}\left(\Gamma_{2}\right)>2 a_{2}+\frac{1}{4 a_{1}}+\frac{1}{2} \\
\lambda_{\text {min }}\left(\Phi_{\chi}-\frac{\Phi_{s}^{T} \Phi_{s}}{4 a_{2}}\right)>\frac{1}{2} \\
\frac{1}{T}=b+\frac{1}{4 a_{1}} \\
\lambda_{\text {min }}(q)>\mu
\end{gathered}
$$

For nonlinear kinematic equation (1) and dynamic equation (2) for the three degrees of freedom DP vessel, under Assumptions 1and 2, the complex disturbances of the DP vessel were estimated using equation (6) for the designed UDE. The first-order filter of equation (25) was designed to avoid the differential explosion. Considering the actual propulsion module inherent structure constraints, the ADS of equation (29) was used to compensate the constraints problem, and the smooth switch function of equation (30) was employed to handle singularity phenomena with the ADS. All the signals of the DP closed-loop control system could be uniformly ultimately bounded by suitably adjusting control parameters $\Gamma_{1}, \Gamma_{2}, T, \Phi_{\chi}, \Phi_{s}, q$ until equations (46), (47), (48), (49), and (50) were satisfied.

Proof: Integrating inequality (46) yields the following:

$$
0 \leq V(t) \leq \frac{\theta}{2 \kappa}+\left(V(0)-\frac{\theta}{2 \kappa}\right) e^{-2 \kappa t}
$$

Equation (51) obviously shows that $V(t)$ is uniformly ultimately bounded. Based on equation (33), signals $\vartheta_{1}, \vartheta_{2}, \delta, \chi, \widetilde{U}_{d}$ are uniformly ultimately bounded. Therefore, we

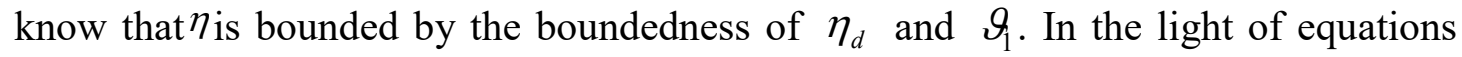
(24), (26), and (28), $\alpha_{1}, v_{d}, v$ are also bounded. Further, it can be determined from the boundedness of $U_{d}$ that the estimation of $\hat{U}_{d}$ is bounded. Thus, all the signals of the DP closed-loop control system are uniformly ultimately bounded. 
Synthesising equations (33) and (51) gives the following:

$$
0 \leq\left\|\vartheta_{1}\right\| \leq \sqrt{\frac{\theta}{\kappa}+2\left[V(0)-\frac{\theta}{2 \kappa}\right] e^{-2 \kappa t}}
$$

Obviously, it can be seen from equation (52) that for any $\kappa_{\vartheta_{1}} \geq \sqrt{\frac{\theta}{\kappa}}$, there exists a time constant $t>T_{\vartheta_{1}}$, such that $\left\|\vartheta_{1}\right\| \leq \kappa_{\vartheta_{1}}$ holds for all $t>T_{\vartheta_{1}}$.In other words, the position and heading error vector $\vartheta_{1}$ converge to the compact set $\Omega_{\vartheta_{1}}=\left\{\vartheta_{1} \in R^{3} \mid\left\|\vartheta_{1}\right\| \leq \kappa_{\vartheta_{1}}\right\}$, which can be made arbitrarily small by appropriately designing the control parameters so that equations (46)-(50) are satisfied. Hence, the vessel can be positioned at the given position and heading $\eta_{d}=\left[\begin{array}{lll}x_{d} & y_{d} & \psi_{d}\end{array}\right]^{T}$ with an arbitrary error precision.

Remark 6: For DP vessels operating in marine environment, it is particularly necessary to improve operational accuracy by estimating complex disturbances. The PID and NLPID controllers don't have advantages when estimating complex disturbances as they don't have disturbance observers. Compared with NN, the UDE mainly deals with modelling uncertainty and disturbances, while the NN only handles modelling uncertainty and has no capability to approximate the disturbance. Thus, the robustness of UDE is stronger than NN estimator. The UDE is an observer constructed based on the internal model principle [29] and embedded in the control system. The $\mathrm{NN}$ is an estimator whose approximation ability needs to be self-adjusted by adjusting weight and multiplying activation function. From this point of view, the UDE is much easier to implement. In general, the UDE-based DSC method is robust and easy to be applied in DPS.

Remark 7: The method of NN-based DSC is one of the state-of-the-art methods, which was employed to the control of marine vessels. NN is usually utilized to estimate the modelling uncertainties so that NN-based DSC has a relatively better performance [38, 39]. Thus, NN-based DSC 
method was also included in several recent references for comparisons. [40-42].

\section{Simulation experiments}

In order to evaluate the effectiveness of the design control law, the supply ship discussed in [43] was taken as the simulation object for simulation experiments. The kinetic model parameters $M, C(v), D, \Delta_{f}$ of the supply ship were given in [44]. The magnitude and rate limits of the forces or moments provided by the propeller are listed in Table 1 [45]. The UDEbased DSC law proposed here is compared with that the NN-based DSC technology [46], the PID control law [25] and the NLPID control method [47] under the same initial conditions and the same sea conditions.

The NN-based DSC law is chosen as follows.

$$
\begin{gathered}
\tau_{N N-D S C}=C(v) v+D v+M \frac{\alpha_{1}-v_{d}}{T}+\hat{W}^{T} h(v)-\Gamma_{4} \vartheta_{2}-\Xi \hat{\varsigma} \\
\dot{\hat{\varsigma}}=P\left[\Xi \vartheta_{2}-\Lambda\left(\hat{\varsigma}-\varsigma^{0}\right)\right] \\
\Xi\left(\vartheta_{2}\right)=\operatorname{diag}\left(\tanh \left(\vartheta_{2,1} / \omega_{1}\right), \tanh \left(\vartheta_{2,2} / \omega_{2}\right) \tanh \left(\vartheta_{2,3} / \omega_{3}\right)\right)
\end{gathered}
$$

where $\omega_{1}=1, \omega_{2}=1, \omega_{3}=0.01, P=\operatorname{diag}\left(10^{4}, 10^{5}, 10^{5}\right), \Lambda=\operatorname{diag}\left(10^{-7}, 10^{-7}, 10^{-9}\right)$, $\varsigma^{0}=\left[\begin{array}{lll}0.1 & 0.1 & 0.1\end{array}\right]^{T}, \quad \Gamma_{4}=\operatorname{diag}\left(9 \times 10^{5}, 9 \times 10^{5}, \quad 1 \times 10^{9}\right), \hat{W} \in R^{63 \times 3}$ is the estimation matrix of the ideal weight vector, $h(v) \in R^{183}$ is the vector of the RBF.

The PID control law is chosen as follows.

$$
\tau_{P I D}=K_{p} \vartheta_{1}(t)+K_{I} \int \vartheta_{1}(t) d t+K_{D} \dot{\vartheta}_{1}(t)
$$

where $K_{P}=\operatorname{diag}\left(1.0 \times 10^{5}, 1.2 \times 10^{5}, 1.5 \times 10^{8}\right), K_{I}=\operatorname{diag}(14,14,14)$, $K_{D}=\operatorname{diag}\left(1.8 \times 10^{6}, 1.8 \times 10^{6}, 1.8 \times 10^{9}\right)$.

The NLPID control law is chosen as follows. 


$$
\tau_{N L P I D}=f\left(\vartheta_{1}\right)\left[K_{p} \vartheta_{1}(t)+K_{I} \int \vartheta_{1}(t) d t+K_{D} \dot{\vartheta}_{1}(t)\right]
$$

where $f\left(\vartheta_{1}\right)=\operatorname{diag}\left(f\left(\vartheta_{1,1}\right) \quad f\left(\vartheta_{1,2}\right) \quad f\left(\vartheta_{1,3}\right)\right)$

$$
\begin{aligned}
f\left(\theta_{1, i}\right) & =\frac{\exp \left(k_{i} \vartheta_{1 i}\right)+\exp \left(-k_{i} \vartheta_{1, i}\right)}{2} \quad i=1,2,3 \\
\vartheta_{1, i} & =\left\{\begin{array}{cc}
\vartheta_{1, i} & \left|\vartheta_{1, i}\right| \leq \vartheta_{1, i M} \\
\vartheta_{1, i M} \operatorname{sgn}\left(\vartheta_{1, i}\right) & \left|\vartheta_{1, i}\right|>\vartheta_{1, i M}
\end{array}\right.
\end{aligned}
$$

where $k_{1}=0.15, k_{2}=0.12, k_{3}=0.15, \vartheta_{1,1 M}=15, \vartheta_{1,2 M}=15, \vartheta_{1,3 M}=\frac{\pi}{36}$.

Table1. Input constraints of supply ship.

\begin{tabular}{lll}
\hline & Magnitude limits & Rate limits \\
\hline In surge & $\tau_{\mathrm{M} 1}=3.76815 \times 10^{2} \mathrm{KN}$ & $\tau_{\mathrm{R} 1}=53.8307 \mathrm{KN}$ \\
In sway & $\tau_{\mathrm{M} 2}=6.80725 \times 10^{2} \mathrm{KN}$ & $\tau_{\mathrm{R} 2}=97.2464 \mathrm{KN}$ \\
In yaw & $\tau_{\mathrm{M} 2}=7.3119 \times 10^{3} \mathrm{KN}$ & $\tau_{\mathrm{R} 3}=1044.6 \mathrm{KN}$ \\
\hline
\end{tabular}

\subsection{Performance of proposed UDE-based DSC method}

This subsection shows how the proposed UDE-based DPS control law was simulated with different disturbances and limitations conditions.

The environment disturbances are described as follows [48]:

$$
\left\{\begin{array}{l}
d(t)=J^{T}(\psi) b \\
\dot{b}=-T_{c}^{-1} b+\zeta \varpi
\end{array}\right.
$$

where $b \in R^{3}$ is the disturbances vector under the earth-fixed coordinate system; $T_{c} \in R^{3 \times 3}$ denotes the time-constant diagonal matrix; $\varpi \in R^{3}$ is a zero-mean Gaussian white noise vector; and $\zeta \in R^{3 \times 3}$ is the diagonal amplitude matrix of $\varpi$. 
Table2. Positioning settling times and error rates of four control laws under two different conditions.

\begin{tabular}{|l|l|l|l|l|l|l|l|l|}
\hline \multirow{2}{*}{$\begin{array}{l}\text { Control law } \\
\text { Case }\end{array}$} & \multicolumn{2}{|l|}{ DSC+UDE } & \multicolumn{2}{l|}{ DSC+NN } & \multicolumn{2}{l|}{ PID } & \multicolumn{2}{l|}{ NLPID } \\
\cline { 2 - 9 } & Case1 & Case2 & Case1 & Case2 & Case1 & Case2 & Case1 & Case2 \\
\hline $\begin{array}{l}\text { Settling } \\
\text { time } \mathrm{t}_{\mathrm{s}}(\mathrm{s})\end{array}$ & 100.4 & 102.9 & 157.5 & - & 119.5 & - & 127.2 & - \\
\hline$\frac{\int_{0}^{1200}\left|\vartheta_{1,1}\right| d t}{\vartheta_{1,1}(0) \times t}$ & $2.126 \%$ & $1.988 \%$ & $2.476 \%$ & $2.616 \%$ & $2.478 \%$ & $2.721 \%$ & $2.531 \%$ & $2.853 \%$ \\
\hline$\frac{\int_{0}^{1200}\left|\vartheta_{1,2}\right| d t}{\vartheta_{1,2}(0) \times t}$ & $1.827 \%$ & $1.688 \%$ & $1.885 \%$ & $2.090 \%$ & $2.006 \%$ & $2.413 \%$ & $1.984 \%$ & $3.319 \%$ \\
\hline$\frac{\int_{0}^{1200}\left|\vartheta_{1,3}\right| d t}{\vartheta_{1,3}(0) \times t}$ & $1.171 \%$ & $1.851 \%$ & $1.266 \%$ & $2.965 \%$ & $1.289 \%$ & $2.980 \%$ & $1.282 \%$ & $3.319 \%$ \\
\hline
\end{tabular}

\section{Case 1: With input magnitude constraints only}

The initial value and related parameters of the unknown environmental disturbances are $b(0)=\left[\begin{array}{lll}10 K N & 10 K N & 10 K N m\end{array}\right]^{T}$, $T_{c}=\operatorname{diag}\left(10^{3}, 10^{3}, 10^{3}\right)$, and $\zeta=\operatorname{diag}\left(10^{2}, 10^{2}, 10^{3}\right)$. The desired position and heading are $\eta_{d}=\left[\begin{array}{lll}0 m & 0 m & 0 \mathrm{rad}\end{array}\right]^{T}$. The initial position and heading are $\eta(0)=\left[\begin{array}{lll}30 m & 30 m & \frac{\pi}{18} \mathrm{rad}\end{array}\right]^{T} \quad$.The $\quad$ initial $\quad$ velocities are $v(0)=\left[\begin{array}{lll}0 m / s & 0 m / s & 0 \mathrm{rad} / \mathrm{s}\end{array}\right]^{T}$. The control parameters are designed as follows: $\Gamma_{1}=\operatorname{diag}(0.15,0.15,0.11), \Gamma_{2}=\operatorname{diag}\left(6 \times 10^{5}, 9 \times 10^{5}, 8 \times 10^{8}\right), q=\operatorname{diag}(2,4,6)$ and $T=0.3$. The initial states of the ADS are $\chi(0)=\left[\begin{array}{lll}3 \times 10^{4} & 3 \times 10^{4} & 3 \times 10^{4}\end{array}\right]^{T}$. The designed parameters are as follows: $\chi_{a i}=5, \chi_{b i}=10, \Phi_{\chi}=\operatorname{diag}(5,5,5)$, $\Phi_{s}=\operatorname{diag}(1,1,1)$ and $\rho=\operatorname{diag}(1,1,1)$.

Under the case that the propulsion module of the DP vessel can provide forces and moments with magnitude constraints, the simulation 
results of the UED-based DSC strategy method, NN-based DSC method, PID control law and NLPID control law are shown in Fig.3 (a)-(e). The control effects of the four methods are intuitively evaluated using the positioning error rates $\frac{\int_{0}^{1200}\left|\vartheta_{1, i}\right| d t}{\vartheta_{1, i}(0) \times t}$ and settling time, which are summarised in Table 2. From Fig.3 (a),(b) and Table 2 it can be seen that four methods have rapid responses and the DP vessel can be kept in the desired position with desired heading. It can be seen from Fig.3(c) and (d) that the great output rate of thrusts is required from about $0 \mathrm{~s} \sim 30 \mathrm{~s}$. However, it is difficult for the output rate of thrusters to meet the requirements. From Fig.3 (e), it can be seen that the complex disturbances are appropriately estimated by UDE. The UDE-based DSC method has a slightly superior performance.

\section{Case 2: With input magnitude and rate constraints}

The input rate limitations are taken into account in this case. The amplitude matrix $\zeta=\operatorname{diag}\left(5 \times 10^{3} \quad 5 \times 10^{3} \quad 5 \times 10^{4}\right)$ of $\varpi$ is increased to 50 times of that in Case 1. The other conditions are consistent with those of Case 1.

The experimental results of the four methods are illustrated in Fig.4 (a)-(f). The performance indexes of the position error rates are also presented to intuitively evaluate the control effects of the four strategies in Table 2. It is observed from Fig.4 (a)-(c) and Table 2 that the DP vessel can't be kept in the desired position with desired heading used PID, NLPID or NN-based DSC method. However, the UDE-based DSC strategy exhibits almost the same performance as that in Case 1. From Fig. 4 (d) and (e), it can be seen that the four methods are limited by the magnitude and rate constraints of the forces and moments. The control inputs and their rates are within the magnitude and rate constraints. From Fig.3 (f), it can be seen that the UDE has a superior estimated performance for the complex disturbances.

\section{Conclusion}


In this study, a UDE-based DSC method has been proposed for the control of a DP vessel with complex disturbances and the input constraints of magnitude and rate. The ADS is used to handle the problem of input constraints, and a smooth switch function was used to avoid the singularity problem caused by the ADS. The UDE is capable of estimating any complex disturbances without prior knowledge of the vessel's motion state. The simulation results have showed that the proposed control strategy has a superior positioning performance. Moreover, the proposed algorithm design is relatively simple, more consistent with engineering practice. Thus, it is easy to be implemented in a practical DPS.

\section{Acknowledgments}

The authors would like to express appreciation for the financial support provided by the National Natural Science Foundation of China (51809113, 51249006), Fujian Province Science and Technology Department (2019H0019), Fujian Provincal Natural Science Foundation (2018J01494),Fujian Education Department (FBJG20180056, JT180266), and Program for New Century Excellent Talents in Fujian Universities (KB16078). The authors are grateful for the comments raised by the reviewers which helped significantly improve the quality of the paper. The authors are also grateful for the helpful discussions provided by Dr. Xin $\mathrm{Hu}$ from Ludong University, China. 


\section{Appendix A}

Proof of Lemma 1: According to the definition of $h\left(\chi_{i}\right)$.

For continuity:

Considering the point $\chi_{i}=\chi_{a i}$, we can obtain

$\lim _{\chi_{i} \rightarrow \chi_{a i}^{-}} h\left(\chi_{i}\right)=0$

$\lim _{\chi_{i} \rightarrow \chi_{a i}^{+}} h\left(\chi_{i}\right)=1-\cos \left(\frac{\pi}{2} \sin (0)\right)=0$

Since $h\left(\chi_{a i}\right)=0$, so $h\left(\chi_{i}\right)$ is continuous at the point $\chi_{i}=\chi_{a i}$.

In the same way, $h\left(\chi_{i}\right)$ is continuous at the points $\chi_{i}=-\chi_{a i}$ and $\chi_{i}= \pm \chi_{b i}$, so the function $h\left(\chi_{i}\right)$ is a is continuous function for $\chi_{i} \in R$.

For derivation:

Considering the point $\chi_{i}=\chi_{a i}$, we can obtain

$\lim _{\chi_{i} \rightarrow \chi_{a i}^{-}} \dot{h}\left(\chi_{i}\right)=0$

$\lim _{\chi_{i} \rightarrow \chi_{a i}^{+}} \dot{h}\left(\chi_{i}\right)=\lim _{\chi_{i} \rightarrow \chi_{a i}^{+}} \frac{\pi^{2}}{4} \frac{2 \chi_{i}}{\chi_{b i}^{2}-\chi_{a i}^{2}} \sin \left(\frac{\pi}{2} \sin \left(\frac{\pi}{2} \frac{\chi_{i}^{2}-\chi_{a i}^{2}}{\chi_{b i}^{2}-\chi_{a i}^{2}}\right)\right) \cos \left(\frac{\pi}{2} \frac{\chi_{i}^{2}-\chi_{a i}^{2}}{\chi_{b i}^{2}-\chi_{a i}^{2}}\right)=0$

so $h\left(\chi_{i}\right)$ is smoothly at the point $\chi_{i}=\chi_{a i}$.

In the same way, $h\left(\chi_{i}\right)$ is smoothly at the points $\chi_{i}=-\chi_{a i}$ and $\chi_{i}= \pm \chi_{b i}$, so the function $h\left(\chi_{i}\right)$ is a is smoothly function for $\chi_{i} \in R$. The proof is completed. 


\section{References}

[1] Z. Liu, Y. Zhang, X. Yu, and C. Yuan, "Unmanned Surface Vehicles: An Overview of Developments and Challenges," Annual Reviews in Control, vol. 41, pp: 71-93, 2016.

[2] V. Hassani, A. J. Sørensen, A. M. Pascoal, and M. Athans, "Robust Dynamic Positioning of Offshore Vessels Using Mixed- $\mu$ Synthesis Modelling, Design, and Practice," Ocean Engineering, vol. 129, pp: 389-400, 2017.

[3] X. Xiang, C. Yu, L. Lapierre, T. Zhang, and Q. Zhang, "Survey on Fuzzy-logic-based Guidance and Control of Marine Surface Vehicles and Underwater Vehicles," International Journal of Fuzzy Systems, vol. 20, no. 2, pp: 572-586, 2018.

[4] A. J. Sørensen, "A Survey of Dynamic Positioning Control Systems," Annual Reviews in Control, vol. 35, pp: 123-136, 2011.

[5] Z. Peng, J. Wang, D. Wang, and Q. L. Han, "An Overview of Recent Advances in Coordinated Control of Multiple Autonomous Surface Vehicles," IEEE Transactions on Industrial Informatics, 2020, DOI: 10.1109/TII.2020.3004343.

[6] Y. Huang, L. Chen, P. Chen, R. R. Negenborn, and P. H. A. J. M. V. Gelder, "Ship Collision Avoidance Methods: State-of-the-art," Safety Science, vol. 121, pp: 451-473, 2020.

[7] L. Wang, Q. Wu, J. Liu, S. Li, and R. R. Negenborn, "State-of-the Art Research on Motion Control of Maritime Autonomous Surface Ships," Journal of Marine Science and Engineering, vol. 7, no.12, pp: 1-32, 2019.

[8] T. I. Fossen, "Marine Control Systems: Guidance, Navigation and Control of Ship, Rigs and Underwater Vehicles," Trondlheim, Norway: Marine Cybernetics, 2002.

[9] J. G. Balchen, N. A. Jenssen, and S. Saelid, "Dynamic Positioning Using Kalman Filtering and Optimal Control Theory," IFACiIFIP Symposium on Automation in Offshore Oil Field Operation, pp: 183-186, 1976.

[10] J. G. Balchen, N. A. Jenssen, E. Mathisen, and S. Sælid, "A Dynamic Positioning System Based on Kalman Filtering and Optimal Control," Modelling, Identification and Control, vol. 1, no. 3, pp: 135-163, 1980.

[11] S. Saelid, N. A. Jenssen, and J. G. Balchen, "Design and Analysis of a Dynamic Positioning 
System Based on Kalman Filtering and Optimal Control," IEEE Transactions on Automatic Control, vol. 28, no. 3, pp: 331-339, 1983.

[12] T. I. Fossen, and T. Perez, "Kalman Filtering for Positioning and Heading Control of Ships and Offshore Rigs," IEEE Control Systems Magazine, vol. 29, no. 6, pp: 32-46, 2009.

[13] H. Ashrafiuon, K. R. Muske, L. C. Mcninch, and R. A. Soltan, "Sliding-mode Tracking Control of Surface Vessels," IEEE Transactions on Industrial Electronics, vol. 55, no. 11, pp: 4004-4012, 2008.

[14] M. Movahhed, S. Dadashi, and M. Danesh, "Adaptive Sliding Mode Control for Autonomous Surface Vessel," Proceedings of the 2011 IEEE International Conference on Mechatronics, Istanbul, Turkey, pp: 522-527, 2011.

[15] Z. Piao, C. Guo, and S. Sun, “Adaptive Backstepping Sliding Mode Dynamic Positioning System for Pod Driven Unmanned Surface Vessel Based on Cerebellar Model Articulation Controller," IEEE Access, vol. 8, pp: 48314-48324, 2020.

[16] J. Velagic, Z. Vukic, and E. Omerdic, “Adaptive Fuzzy Ship Autopilot for Track-keeping," Control Engineering Practice, vol. 11, pp: 433-443, 2003.

[17] X. H, J. Du, and J. Shi, “Adaptive Fuzzy Controller Design for Dynamic Positioning System of Vessels," Applied Ocean Research, vol. 53, pp: 46-53, 2015.

[18] R. Skulstad, G. Li, H. Zhang, and T.I. Fossen, "A Neural Network Approach to Control Allocation of Ships for Dynamic Positioning," IFAC PapersOnline, vol. 51, no. 29, pp: 128-133, 2018.

[19] Z. Zhao, W. He, and S. S. Ge, "Adaptive Neural Network Control of A Fully Actuated Marine Surface Vessel with Multiple Output Constraints," IEEE Transactions on Control Systems Technology, vol. 22, no. 4, pp: 1536-1543, 2014.

[20] Z. Lei, C. Guo, and Y. Fan, "Dynamic Positioning System Based on Active Disturbance Rejection Technology". Journal of Ocean University of China, vol. 14, no. 4, pp: 636-644, 2015.

[21] D. Wu, F. Ren, L. Qiao, and W. Zhang, “Active Disturbance Rejection Controller Design for Dynamically Positioned Vessels Based on Adaptive Hybrid Biogeography-Based Optimization and Differential Evolution,” ISA Transactions, vol. 78, pp: 56-65, 2018.

[22] X. Hu, J. Du, and Y. Sun, "Robust Adaptive Control for Dynamic Positioning of Ships," 
IEEE Journal of Oceanic Engineering, vol.42, no.4, pp: 826-835, 2017.

[23] J. Du, Y. Yang, D. Wang, and C. Guo, "A Robust Adaptive Neural Networks Controller for Maritime Dynamic Positioning System," Neurocomputing, vol. 110, no. 13, pp: 128-136, 2013.

[24] Z. Chen, Y. Zhang, Y. Nie, J. Tang, and S. Zhu, “Adaptive Sliding Mode Control Design for Nonlinear Unmanned Surface Vessel Using RBFNN and Disturbance Observer," IEEE Access, vol. 8, pp: 45457-45467, 2020.

[25] J. Du, X. Hu, M. Krstić, and Y. Sun, "Robust Dynamic Positioning of Ships with Disturbances under Input Saturation,” Automatica, vol. 73, pp: 207-214, 2016.

[26] X. Hu, J. Du, G. Zhu, and Y. Sun, "Robust Adaptive NN Control of Dynamically Positioned Vessels under Input Constraints,” Neurocomputing, vol. 318, pp: 201-212, 2018.

[27] Y. Ma, G. Zhu, and Z. Li, "Error-Driven-Based Nonlinear Feedback Recursive Design for Adaptive NN Trajectory Tracking Control of Surface Ships with Input Saturation," IEEE Intelligent Transportation Systems Magazine, vol. 11, no. 2, pp: 17-27,2019.

[28] Q. C. Zhong, and D. Rees, "Control of Uncertain LTI Systems Based on an Uncertainty and Disturbance Estimator.” Journal of Dynamic Systems Measurement and Control, vol. 126, pp: 905-910, 2004.

[29] B. Ren, Q. C. Zhong, and J. Dai, “Asymptotic Reference Tracking and Disturbance Rejection of UDE-Based Robust Control," IEEE Transactions on Industrial Electronics, vol. 64, no. 4, pp: 3166-3176, 2017.

[30] L. Sun, D. Li, Q. C. Zhong, and K. Y. Lee, "Control of a Class of Industrial Processes With Time Delay Based on a Modified Uncertainty and Disturbance Estimator," IEEE Transactions on Industrial Electronics, vol. 63, no. 11, pp: 7018-7028, 2017.

[31] Z. Tian, Q. C. Zhong, B. Ren, and J. Yuan, "Stabilisability Analysis and Design of UDE-Based Robust Control," IET Control Theory \& Applications, vol. 13, no. 10, pp: 1445-1453, 2019.

[32] T. I. Fossen, "Handbook of Marine Craft Hydrodynamics and Motion Control," Chichester: John Wiley and Sons, 2011.

[33] X. Hu, and J. Du, "Robust Nonlinear Control Design for Dynamic Positioning of Marine Vessels With Thruster System Dynamics,” Nonlinear Dynamics, vol. 94, pp: 365-376, 2018. 
[34] I. Aharon, D. Shmilovitz, and A. Kuperman, "Uncertainty and Disturbance Estimator-Based Controllers Design Under Finite Control Bandwidth Constraint," IEEE Transactions on Industrial Electronics, vol. 65, no. 2, pp: 1439-1449, 2018.

[35] M. Chen, S. S. Ge, and B. Ren, "Adaptive Tracking Control of Uncertain MIMO Nonlinear Systems with Input Constraints," Automatica, vol. 47, no. 3, pp: 452-465, 2011.

[36] Z. Zheng, and M. Feroskhan, "Path Following of a Surface Vessel With Prescribed Performance in the Presence of Input Saturation and External Disturbances," IEEE/ASME Transactions on Mechatronics, vol. 22, no. 6, pp: 2564 -2575, 2017.

[37] J. Wu, W. Chen, D. Zhao, and J. Li, "Globally Stable Direct Adaptive Backstepping NN Control for Uncertain Nonlinear Strict-Feedback Systems," Neurocomputing, vol. 122, pp: 134-147, 2013.

[38] G. Zhu, J. Du, and Y. Kao, "Robust Adaptive Neural Trajectory Tracking Control of Surface Vessels under Input and Output Constraints," Journal of the Franklin Institute, vol. 357, pp: 2020.

[39] G. Zhang, M. Yao, J. Xu, and W. Zhang, "Robust Neural Event-Triggered Control for Dynamic Positioning Ships With Actuator Faults," Ocean Engineering, vol. 207, pp: 1-12, 2020.

[40] Z. Shen, and X. Zhang, "Recursive Sliding-mode Dynamic Surface Adaptive Control for Ship Trajectory Tracking With Nonlinear Gains," Acta Automatica Sinica, vol. 44, no. 10, pp: 1833-1841, 2018.

[41] K. D. Ellenrueder, "Dynamic Surface Control of Trajectory Tracking Marine Vehicles With Actuator Magnitude and Rate Limits," Automatica, vol. 105, pp: 433-442, 2019.

[42] Y. Deng, X. Zhang, and G. Zhang, "Dynamic Position System of Marine Surface Vessel With ESO and Input Saturation Control," Systems Engineering and Electronics, vol. 41, no. 5, pp: 1110-1107, 2019.

[43] T. I. Fossen,S. I. Sagatun, and A. J. Sǿensen, "Identification of Dynamically Positioned Ships," Model Identification Control, vol. 17, no. 2, pp: 153-165, 1996.

[44] Z. Shen, Y. Wang, H. Yu, and C. Guo, "Finite-time Adaptive Tracking Control of Marine Vehicles With Complex Unknowns and Input Saturation," Ocean Engineering, vol. 198, pp: $1-8,2020$ 
[45] J. Alme, "Autotuned Dynamic Positioning for Marine Surface Vessels," (Master's thesis), Trondheim, Norway: Norwegian University of Science and Technology, pp: 79-80, 2008.

[46] D. Wang, and J. Huang, "Neural Network-Based Adaptive Dynamic Surface Control for a Class of Uncertain Nonlinear Systems in Strict-Feedback Form," IEEE Transactions on Neural Networks, vol. 16, no. 1, pp: 195-202, 2005.

[47] Y. X. Su, D. Sun, and B. Y. Duan, "Design of an Enhanced Nonlinear PID Controller," Mechatronics, vol. 15, pp: 1005-1024, 2005.

[48] T. I. Fossen and J. P. Strand., "Passive Nonlinear Observer Design for Ships Using Lyapunov Methods: Full-scale Experiments With a Supply Vessel,” Automatica, vol. 35, no. 1, pp: 3-16, 1999. 


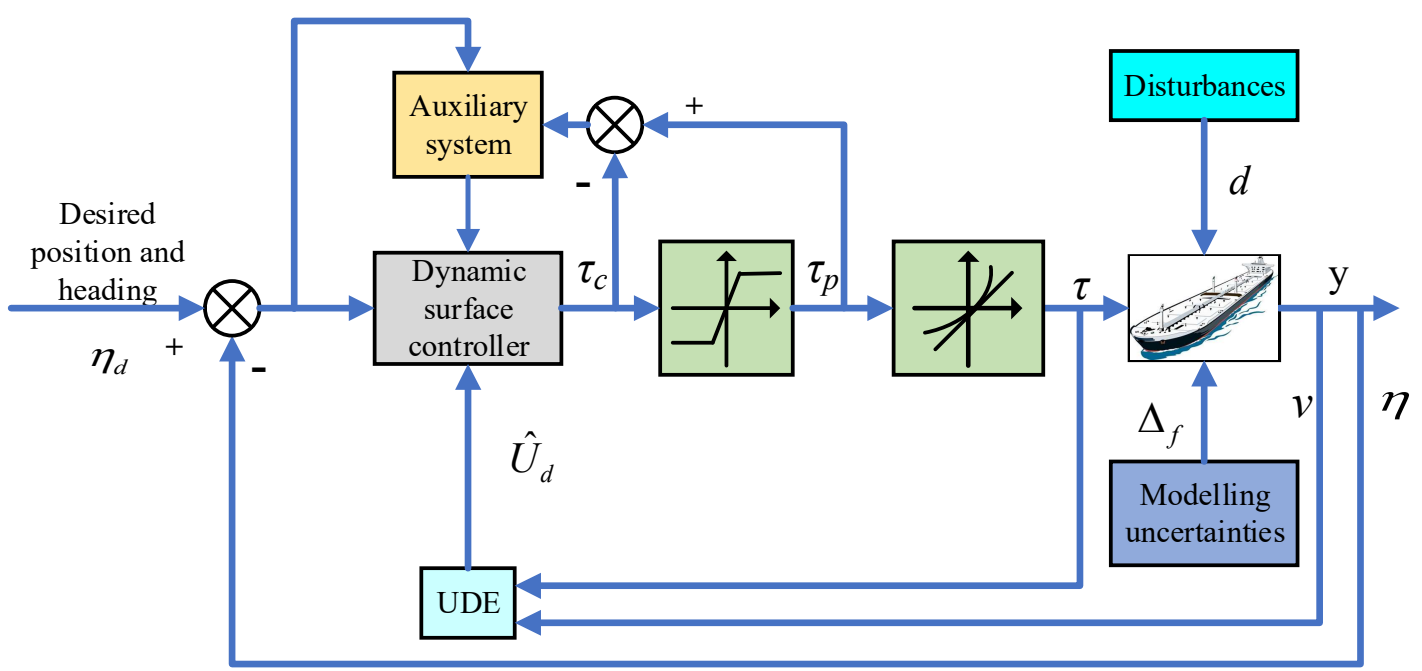

Fig.1. UDE-based dynamic surface controller for vessel

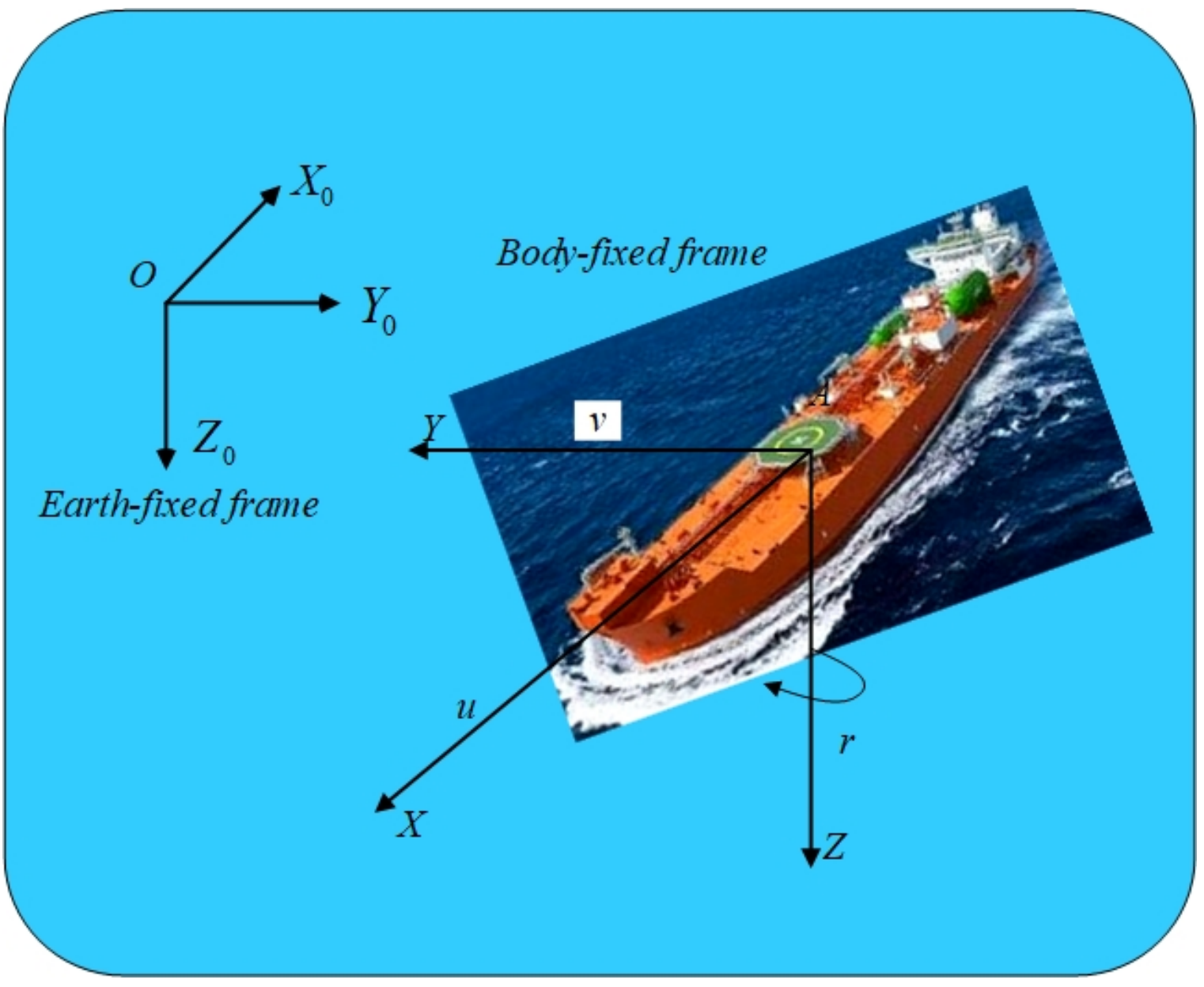

Fig.2. Earth-fixed and body-fixed coordinate frames[32]. 


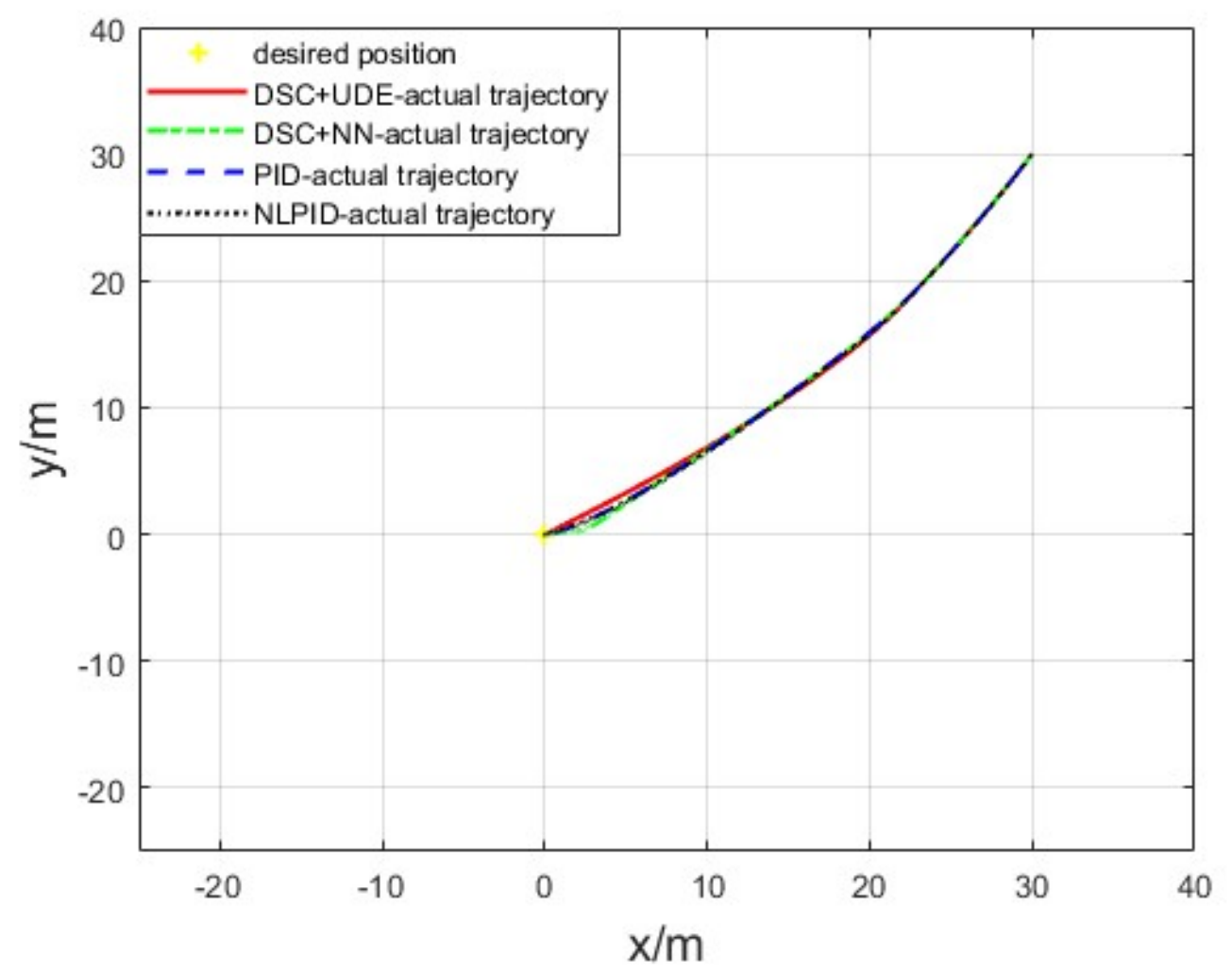

(a). Desired position and actual trajectory of vessel in xy-coordinate frame
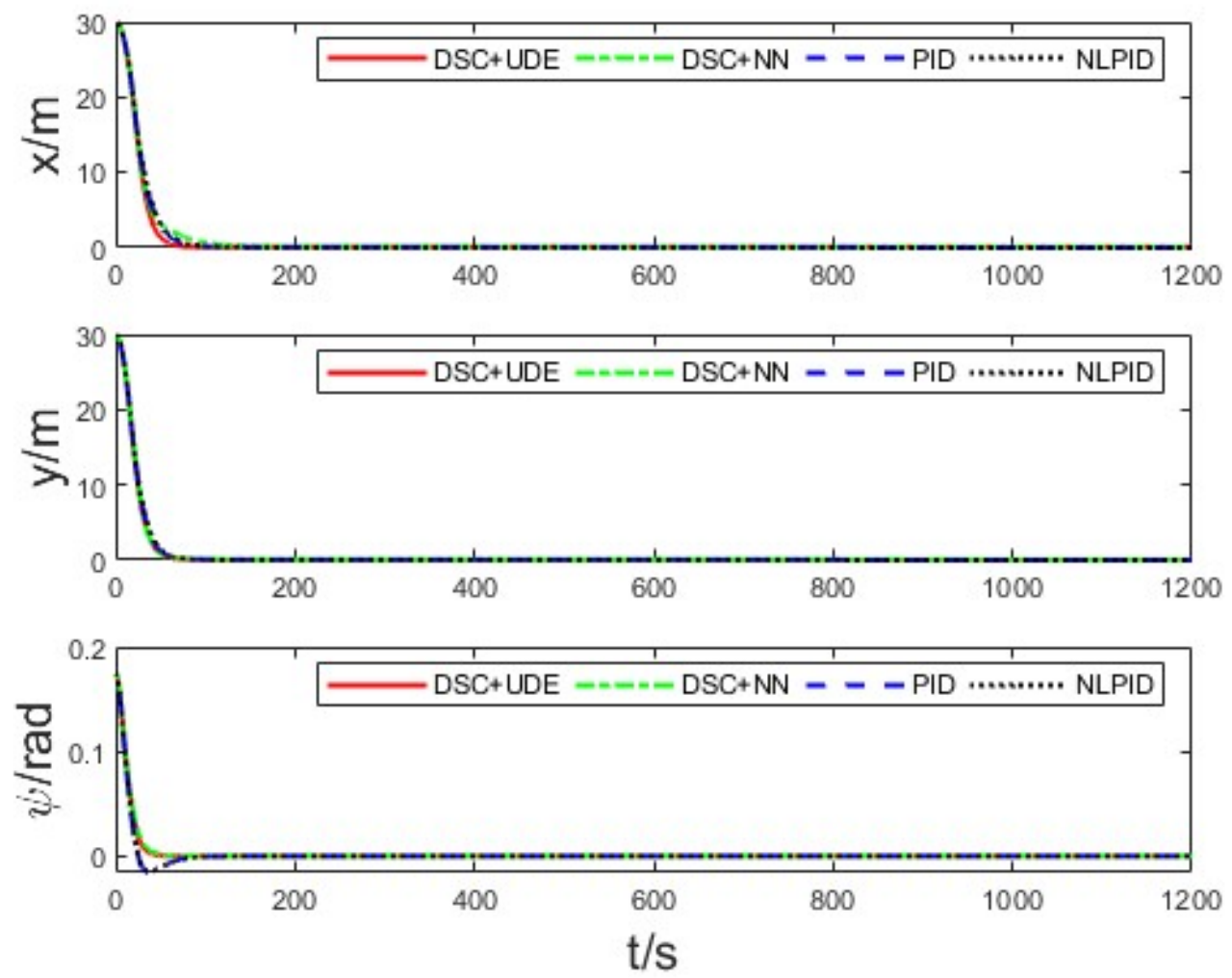

(b). The position and heading of vessel 

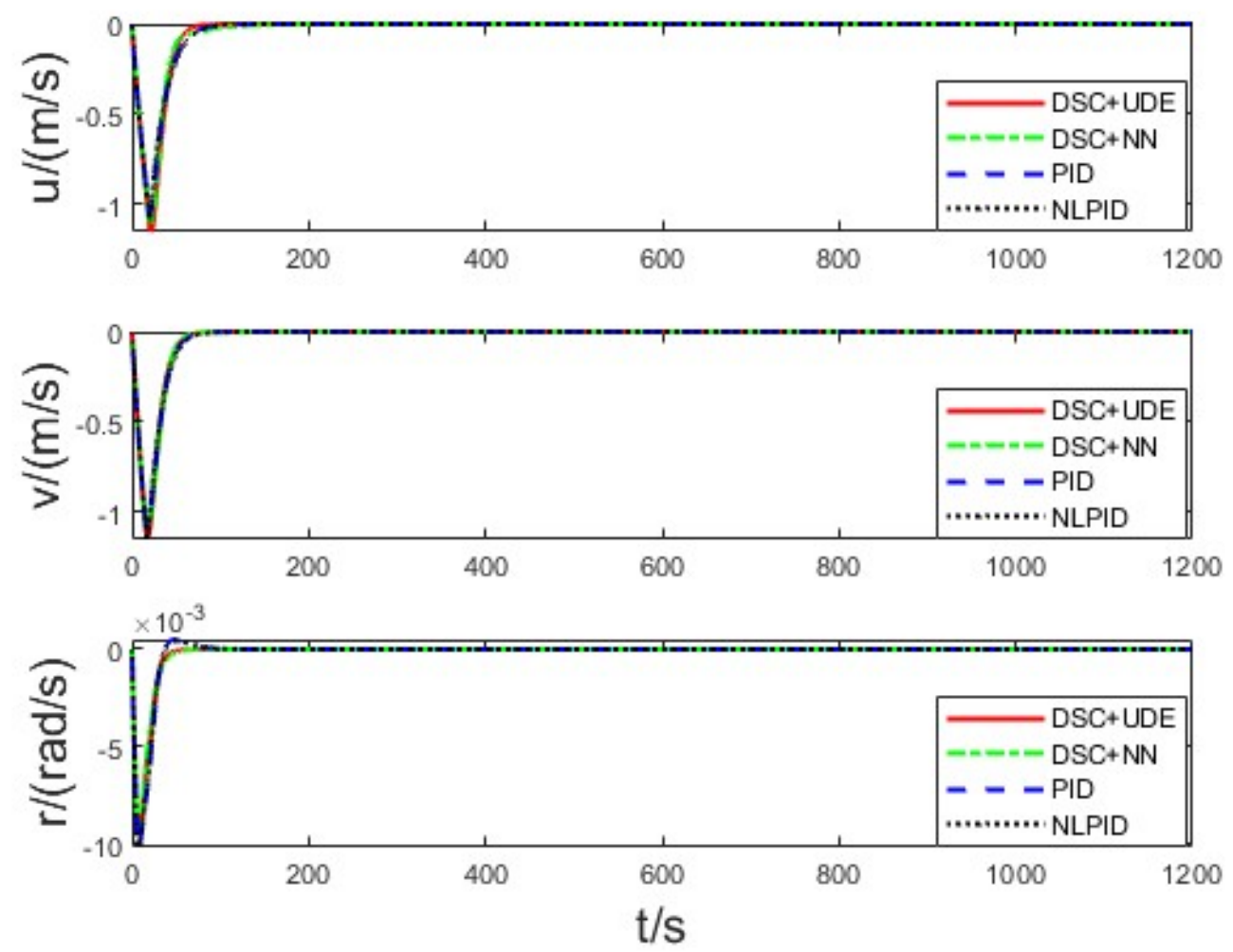

(c). The vessel velocities $(u, v, r)$.
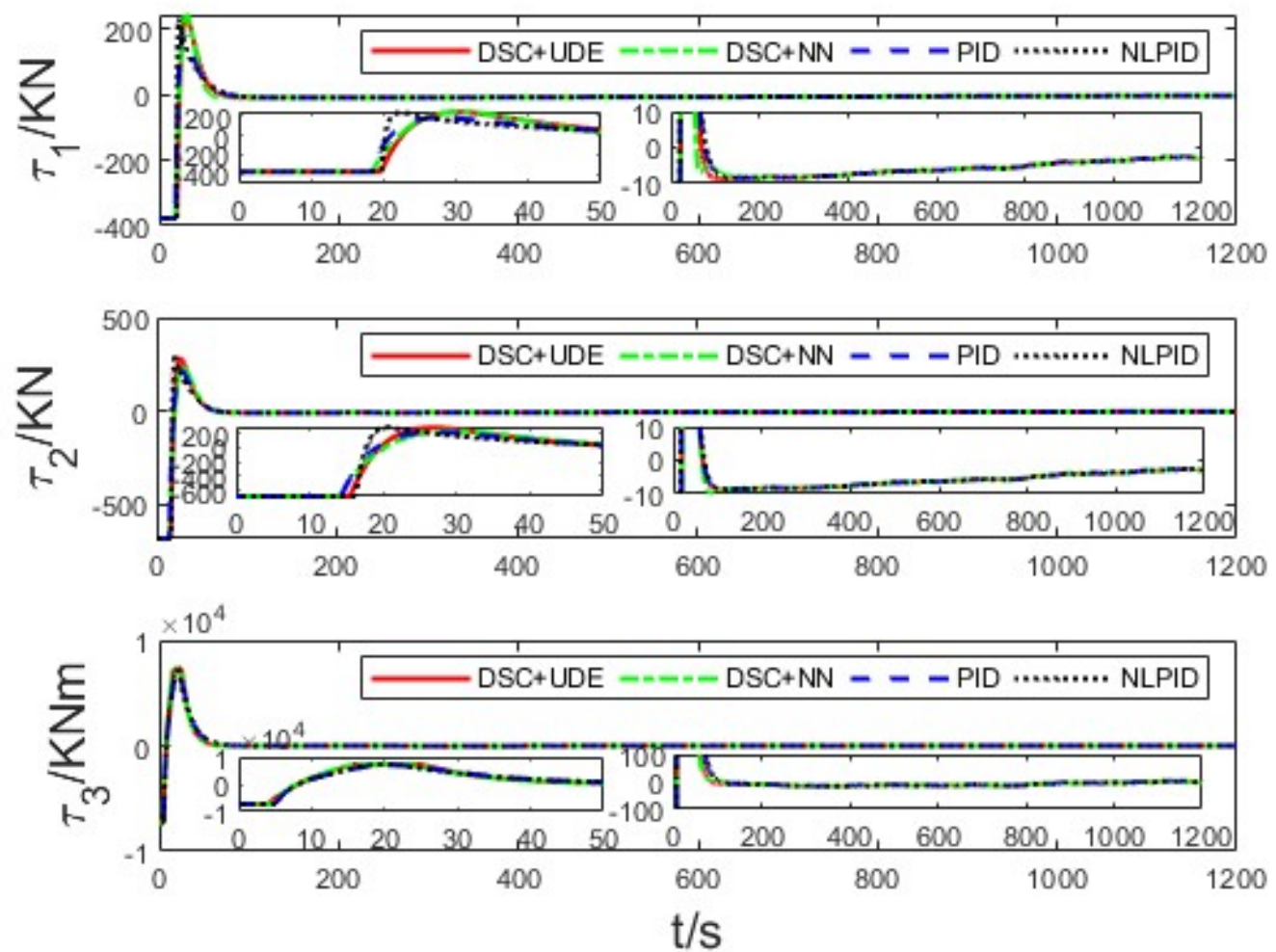

(d). Control forces and moments with magnitude constraints 

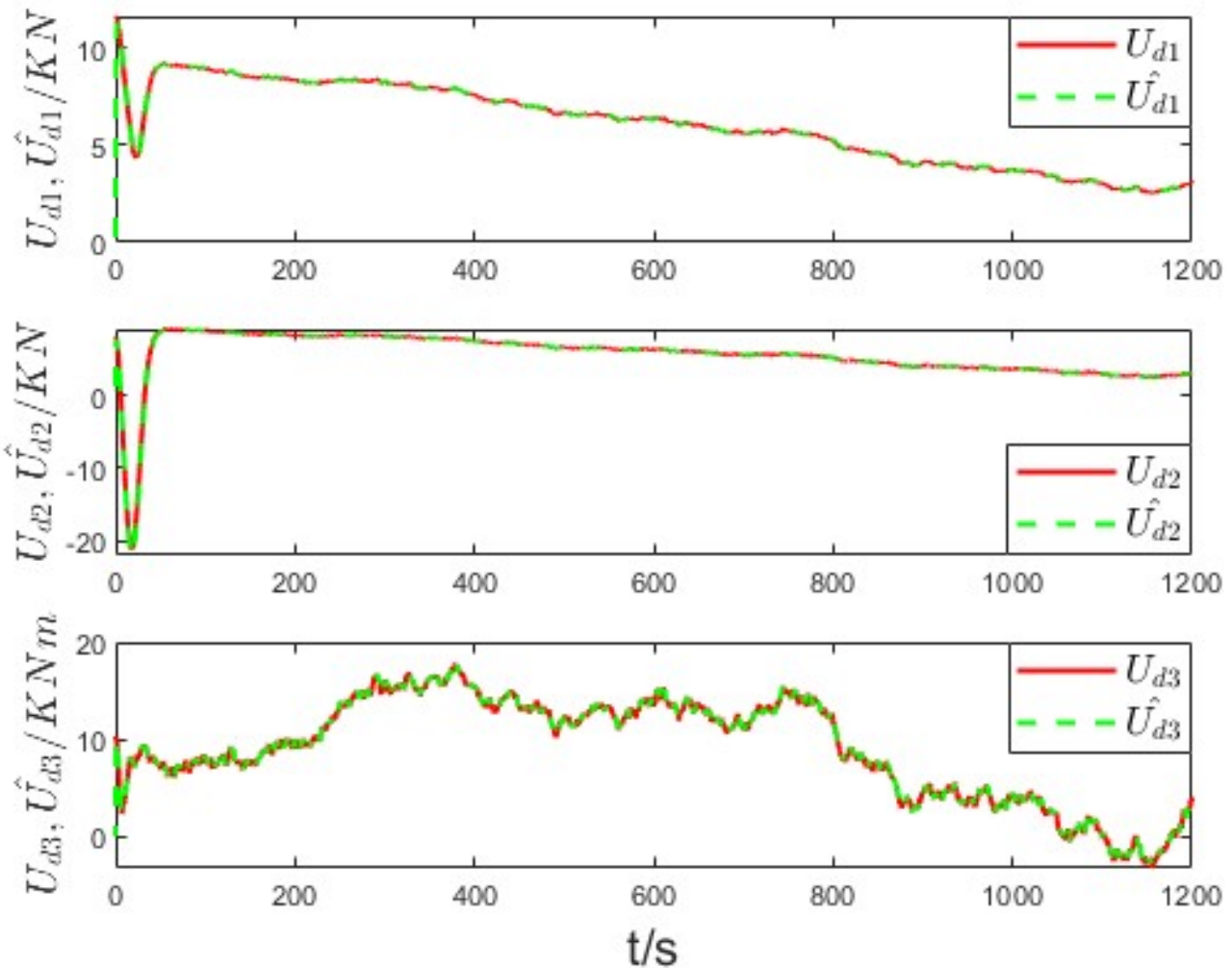

(e). Disturbances and uncertainties of DP vessel and its estimation.

Fig.3.Simulation results with input magnitude limitations. 


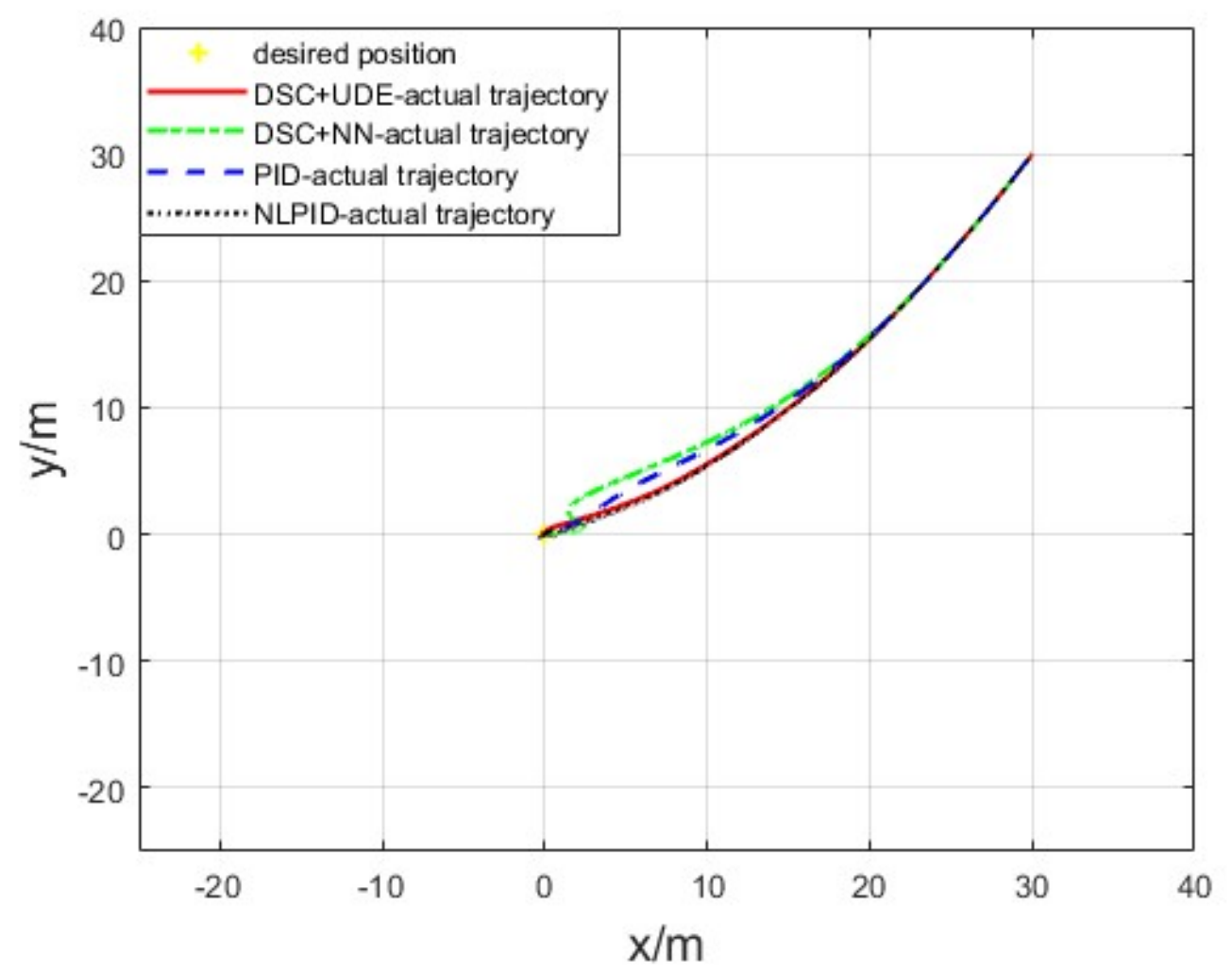

(a). Desired position and actual trajectory of vessel in xy-coordinate frame
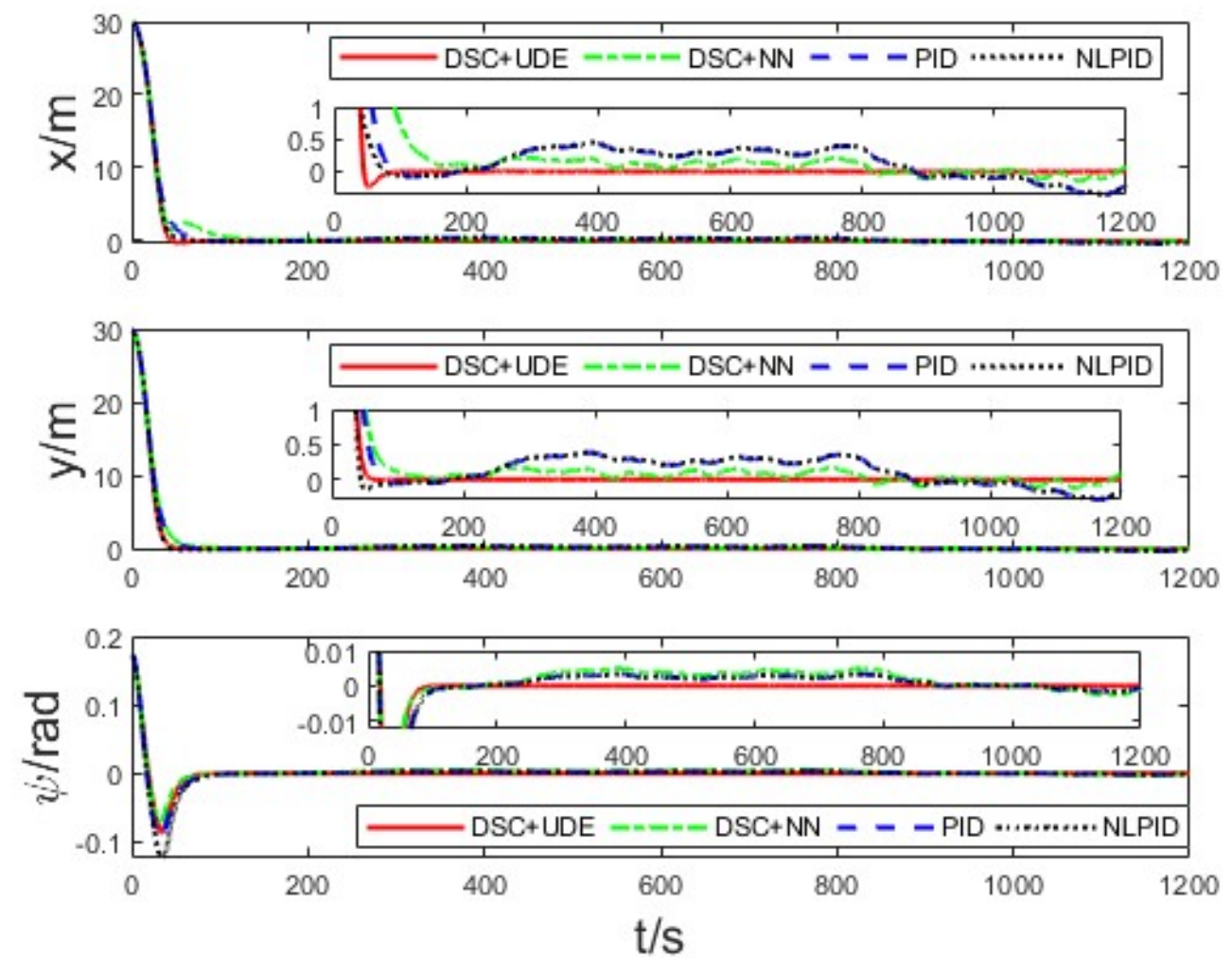

(b). The position and heading of vessel 

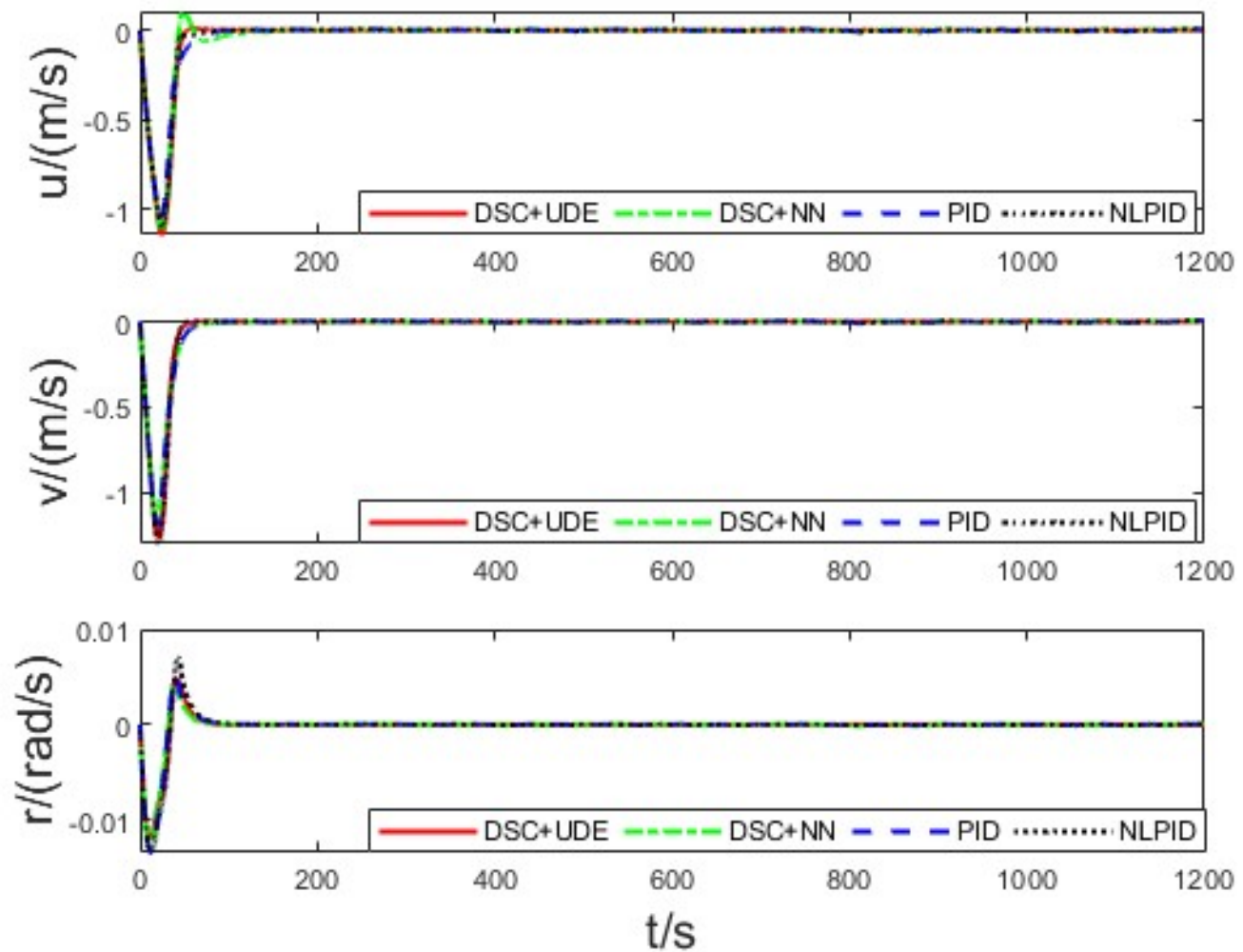

(c). The velocity $(u, v, r)$ of vessel
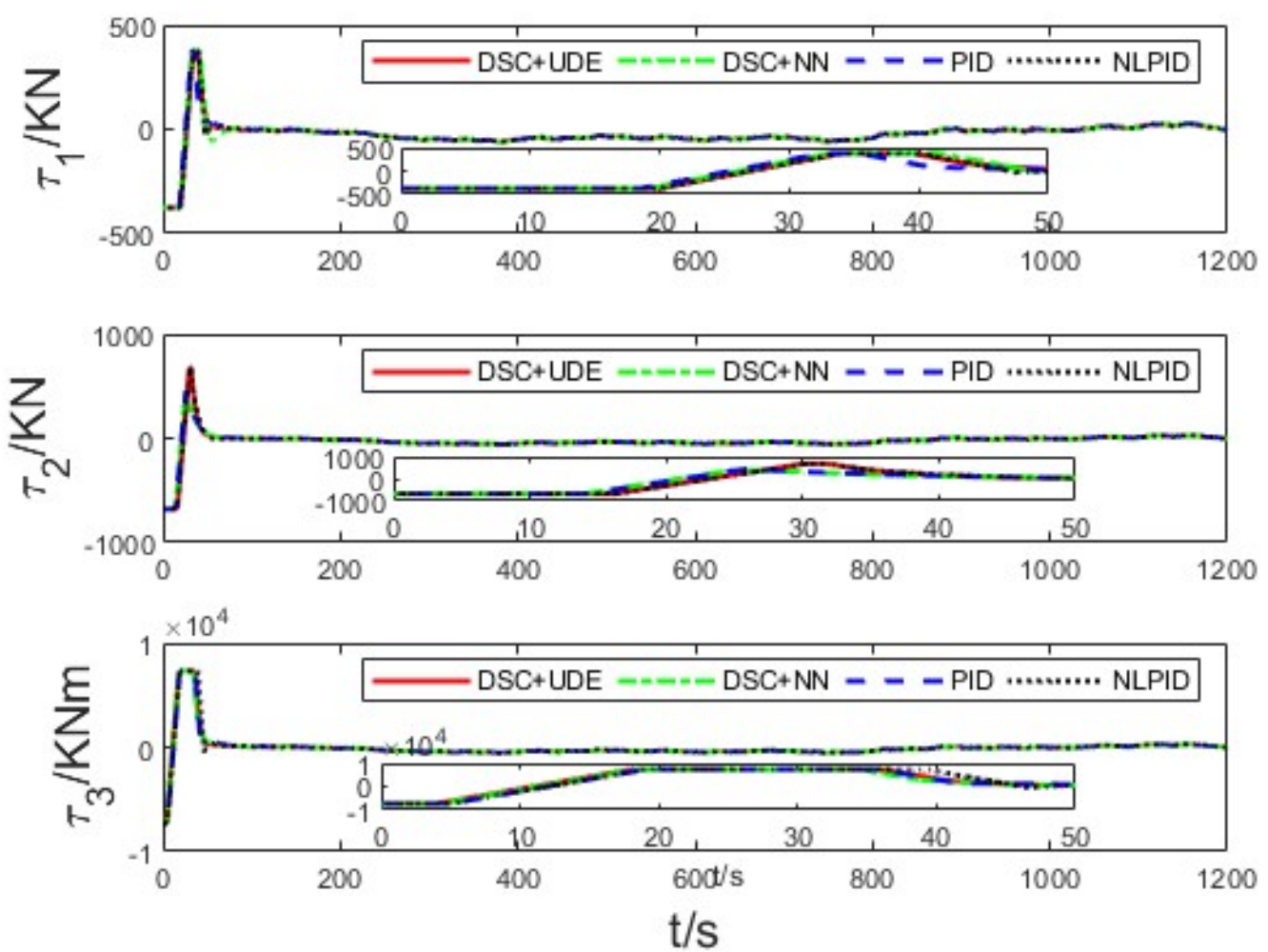

(d).Control forces and moments with magnitude and rate limitations 

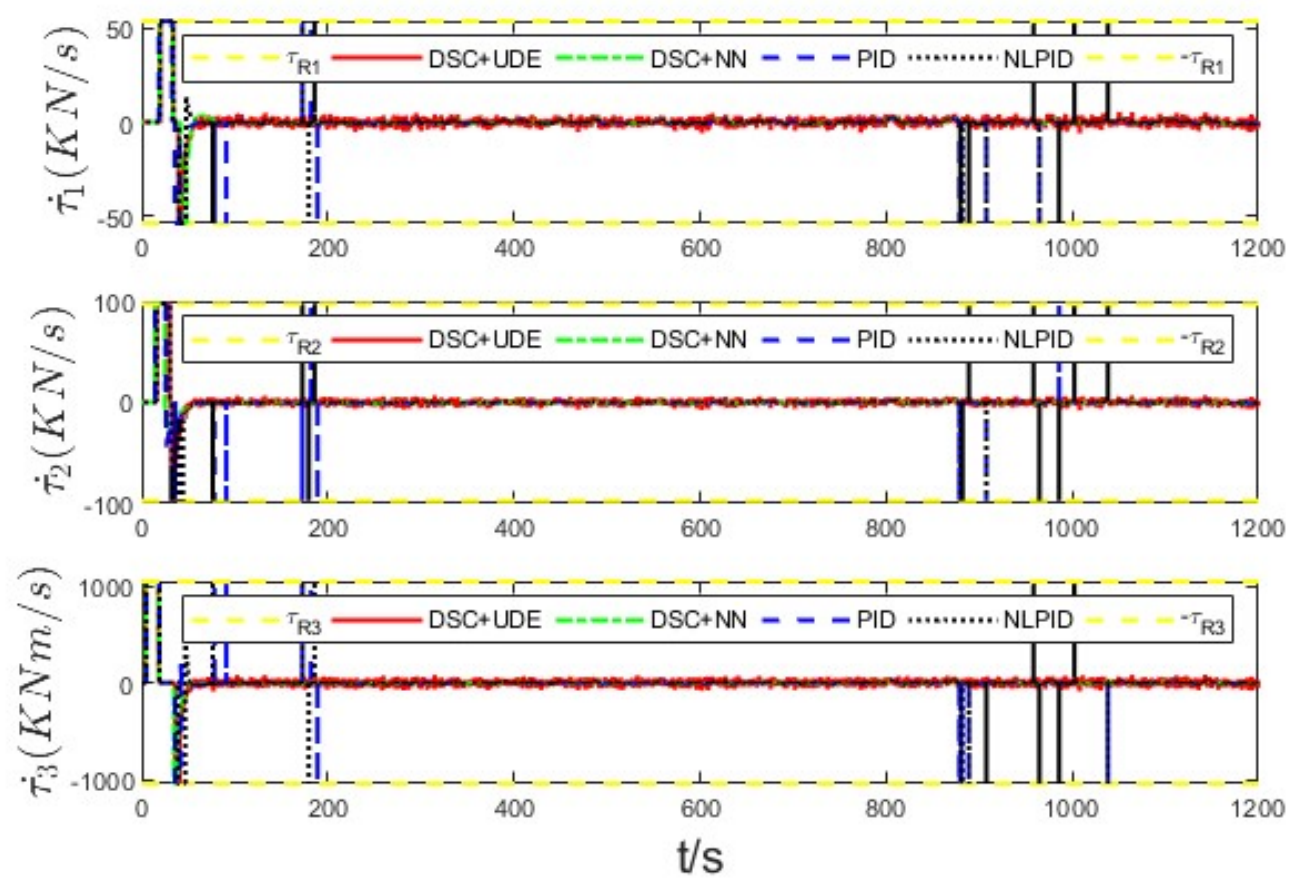

(e) Control input rates $\dot{\tau}_{1}, \dot{\tau}_{2}, \dot{\tau}_{3}$
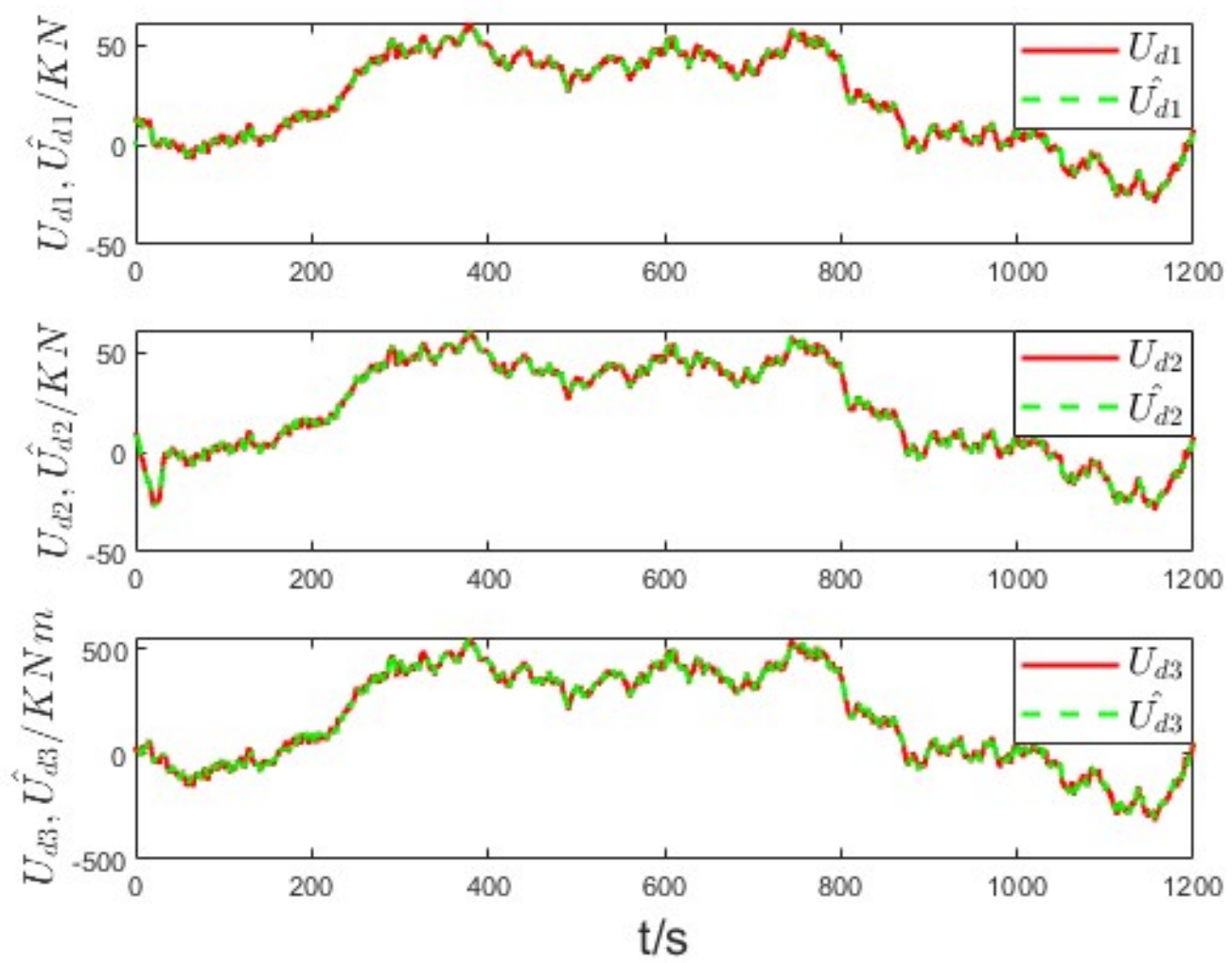

(f). Disturbances and uncertainties of DP vessel and its estimation.

Fig.4.Simulation results with input magnitude and rate constraints. 